\title{
A multidimensional nomogram combining imaging features and clinical factors to predict the invasiveness and metastasis of combined hepatocellular cholangiocarcinoma
}

\author{
Yi Wang ${ }^{1 \#}$, Chang-Wu Zhou ${ }^{1 \#}$, Gui-Qi Zhu ${ }^{2 \#}$, Na Li ${ }^{1}$, Xian-Ling Qian ${ }^{1}$, Huan-Huan Chong ${ }^{1}$, Chun Yang ${ }^{1}$, \\ Meng-Su Zeng' \\ ${ }^{1}$ Department of Radiology, Zhongshan Hospital, Fudan University, Shanghai Institute of Medical Imaging, Shanghai, China; ${ }^{2}$ Department of Liver \\ Surgery and Transplantation, Liver Cancer Institute, Zhongshan Hospital, Fudan University, Shanghai, China \\ Contributions: (I) Conception and design: Y Wang, GQ Zhu, N Li, HH Chong; (II) Administrative support: C Yang, MS Zeng; (III) Provision \\ of study materials or patients: Y Wang, CW Zhou; (IV) Collection and assembly of data: Y Wang, CW Zhou, XL Qian; (V) Data analysis and \\ interpretation: Y Wang, GQ Zhu, N Li; (VI) Manuscript writing: All authors; (VII) Final approval of manuscript: All authors. \\ \#These authors contributed equally to this work. \\ Correspondence to: Meng-Su Zeng, MD, PhD; Chun Yang, MD, PhD. Department of Radiology, Zhongshan Hospital, Fudan University, Shanghai \\ Institute of Medical Imaging, Xuhui District, Shanghai 200032, China. Email: zhudahuaidan@yeah.net; dryangchun@hotmail.com.
}

Background: Combined hepatocellular cholangiocarcinoma (CHCC-CCA) is a rare type of primary liver cancer having aggressive behavior. Few studies have investigated the prognostic factors of CHCC-CCA. Therefore, this study aimed to establish a nomogram to evaluate the risk of microvascular invasion (MVI) and the presence of satellite nodules and lymph node metastasis (LNM), which are associated with prognosis. Methods: One hundred and seventy-one patients pathologically diagnosed with CHCC-CCA were divided into a training set $(n=116)$ and validation set $(n=55)$. Logistic regression analysis was used to assess the relative value of clinical factors associated with the presence of MVI and satellite nodules. The least absolute shrinkage and selection operator (LASSO) algorithm was used to establish the imaging model of all outcomes, and to build clinical model of LNM. Nomograms were constructed by incorporating clinical risk factors and imaging features. The model performance was evaluated on the training and validation sets to determine its discrimination ability, calibration, and clinical utility. Kaplan Meier analysis and time dependent receiver operating characteristic (ROC) were displayed to evaluate the prognosis value of the predicted nomograms of MVI and satellite nodule.

Results: A nomogram comprising the platelet to lymphocyte ratio (PLR), albumin-to-alkaline phosphatase ratio (AAPR) and imaging model was established for the prediction of MVI. Carcinoembryonic antigen (CEA) level and size were combined with the imaging model to establish a nomogram for the prediction of the presence of satellite nodules. Favorable calibration and discrimination were observed in the training and validation sets for the MVI nomogram (C-indexes of 0.857 and 0.795$)$, the nomogram for predicting satellite nodules (C-indexes of 0.919 and 0.883 ) and the LNM nomogram (C-indexes of 0.872 and 0.666). Decision curve analysis (DCA) further confirmed the clinical utility of the nomograms. The preoperatively predicted MVI and satellite nodules by the combined nomograms achieved satisfactory performance in recurrence-free survival (RFS) and overall survival (OS) prediction.

Conclusions: The proposed nomograms incorporating clinical risk factors and imaging features achieved satisfactory performance for individualized preoperative predictions of MVI, the presence of satellite nodules, and LNM. The prediction models were demonstrated to be good indicator for predicting the prognosis of CHCC-CCA, facilitating treatment strategy optimization for patients with CHCC-CCA.

Keywords: Combined hepatocellular cholangiocarcinoma (CHCC-CCA); imaging features; nomogram; microvascular invasion (MVI); prognosis 
Submitted May 15, 2021. Accepted for publication Jul 23, 2021.

doi: 10.21037/atm-21-2500

View this article at: https://dx.doi.org/10.21037/atm-21-2500

\section{Introduction}

Combined hepatocellular cholangiocarcinoma (CHCCCCA) is a rare form of primary liver malignancy that exhibits features of both hepatocytic and cholangiocytic differentiation. CHCC-CCA accounts for approximately $2-5 \%$ of all primary liver cancer cases, and 5 -year survival rate of CHCC-CCA is $17.7 \%(1,2)$. Owing to its low incidence rate, many problems regarding its diagnostic, therapeutic, and prognostic features have not yet been thoroughly understood or defined.

Although there have been substantial studies investigating both preoperative clinical or radiologic variables individually or in combination to predict the prognostic factors of HCC preoperatively, such as microvascular invasion (MVI) and lymph node metastasis (LNM) (3-5), the established models targeting HCC are not suitable for CHCC-CCA since the proportions of hepatocytic and cholangiocytic characteristics in the lesion cannot be determined prior to biopsy or surgery. Notably, imaging features can depict a variety of lesion properties. For example, the Liver Imaging Reporting and Data System (LI-RADS) incorporates imaging features into a standardized categorization system for the detection of hepatic lesions (6). The imaging features of CHCCCCA show considerable variation and can be classified into LR-M or LR-4/LR-5, accounting for 32-63\% and 7-72\%, respectively, according to recent studies. This system was demonstrated to have both diagnostic and prognostic value, with reported studies suggesting that CHCC-CCA patients categorized as LR-M had a worse prognosis than those categorized as LR-4/LR-5 $(7,8)$.

Moreover, whether the clinical or pathological characteristics of CHCC-CCA resemble HCC or ICC is still controversial (9). Some scholars have reported the presence of hepatitis virus infection and elevated $\alpha$-fetoprotein (AFP), similar to HCC, while others have reported serum CA199 elevation and incomplete capsule formation, similar to ICC $(10,11)$. It was also found that CHCC-CCA had the propensity to mimic $\mathrm{HCC}$ with respect to venous invasion and satellite formation, while it resembled ICC with respect to LNM and bile duct involvement (12). Owing to these sophisticated differences, it is crucial to develop a specific model to explore the aggressive biologic behavior associated with the prognosis of CHCC-CCA. To the best of our knowledge, no study has yet combined imaging features with clinical characteristics to predict related prognostic factors. Therefore, the aim of this study was to develop and validate a novel multidimensional nomogram that incorporates both for preoperative prediction of the crucial factors associated with prognosis, including MVI, satellite nodules and LNM, which could facilitate a more individualized treatment approach. The prognostic values of the prediction models were also explored.

We presented the following article in accordance with the TRIPOD reporting checklist (available at https://dx.doi. org/10.21037/atm-21-2500).

\section{Methods}

\section{Patient selection and study design}

The study flow chart for this study is shown in Figure 1A,1B. We retrospectively screened the clinical records of patients with postoperative pathologically confirmed CHCCCCA between January 2015 and December 2019, which were retrieved from the information system of Zhongshan Hospital, Fudan University. All of the patients received curative surgery. CHCC-CCA was confirmed according to pathological diagnosis referring to the $2010 \mathrm{WHO}$ classification system for CHCC-CCA. A total of 146 patients were classified as classical type, 18 patients were classified as typical subtype of stem cell features, five patients were classified as intermediate cell subtype of stem cell features and two patients were classified as cholangiolocellular subtype of stem cell features (13). The inclusion criteria were as follows: (I) patients with clear pathologically confirmed CHCC-CCA after liver resection; (II) Child-Pugh A or B liver function; (III) patients with detailed preoperative laboratory data; and (IV) MRI examinations performed within 30 days before hepatectomy of sufficient quality to satisfy the diagnostic criteria. Additionally, patients were excluded if they met the following exclusion criteria: (I) previous treatment history, such as transarterial chemoembolization (TACE) therapy and neoadjuvant chemotherapy, and (II) synchronous or previous malignancies. Subsequently, 171 CHCC-CCA 
A

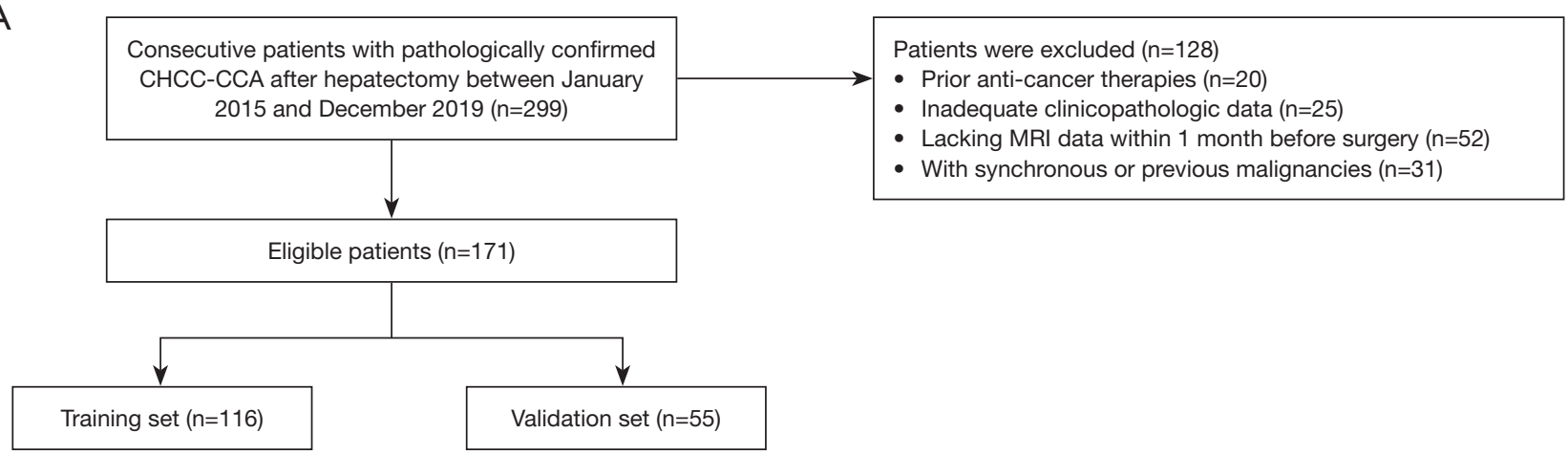

B

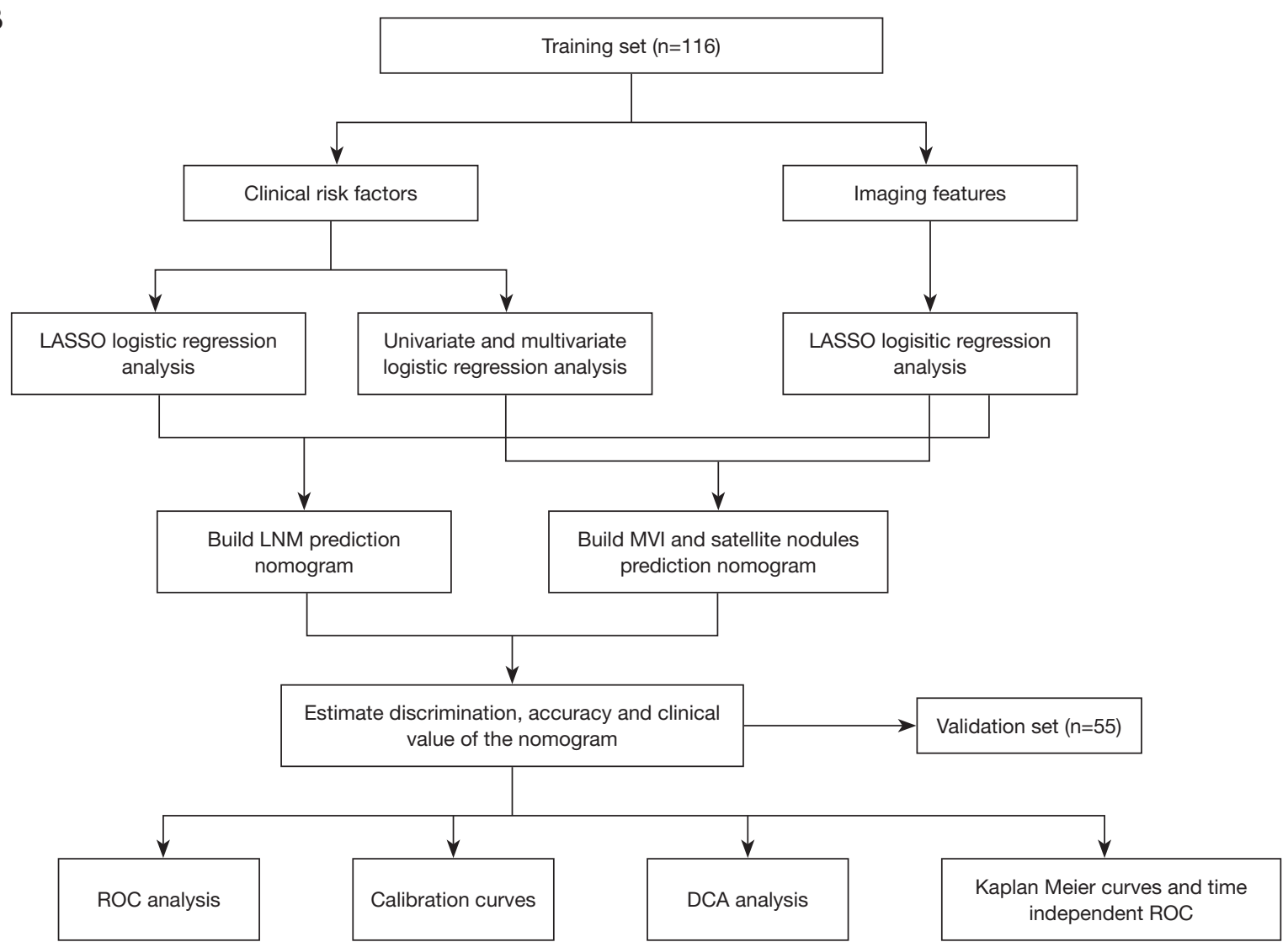

Figure 1 Study flowchart. (A) Flowchart detailing the patient selection process and exclusion criteria; (B) analysis workflow of this study. CHCC-CCA, combined hepatocellular cholangiocarcinoma; LASSO, least absolute shrinkage, and selection operator; LNM, lymph node metastasis; MVI, microvascular invasion; ROC, receiver operating characteristic; DCA, Decision curve analysis.

patients were randomly divided into the training set $(\mathrm{n}=116)$ and validation set ( $\mathrm{n}=55)$ using "Caret" $\mathrm{R}$ package (14). This retrospective study was conducted in accordance with the Declaration of Helsinki (as revised in 2013). The study was approved by the Clinical Research Ethics Committee of Zhongshan Hospital of Fudan University (B2019-139R) and individual consent for this retrospective analysis was waived.

\section{Liver MRI}

All patients were scanned with a 1.5-T MR scanner (Magnetom Aera, Siemens Healthcare). Routine liver 
protocols consisted of transverse T2WI, T1WI in-phase and opposed-phase, and diffusion-weighted imaging (DWI, $\mathrm{b}$ value $=0,50$, and $500 \mathrm{~s} / \mathrm{mm}^{2}$ ). Dynamic imaging was performed with a T1-weighted fat-suppressed sequence. The arterial phase was acquired when the contrast agent (Gd-DTPA, Magnevist, Bayer HealthCare) reached the ascending aorta after the intravenous administration of GdDTPA at a dose of $0.1 \mathrm{mmol} / \mathrm{kg}$ at a rate of $2 \mathrm{~mL} / \mathrm{s}$. The portal venous phase and delayed phase were operated on at 60-70 and 160-180 s, respectively.

\section{Preoperative clinicopathological and imaging variables}

Baseline and clinicopathological variables were collected, including age, sex, etiology, laboratory tests and tumor characteristics (i.e., tumor size, tumor number and tumor location). Receiver operating characteristic (ROC) curves were utilized to determine the optimal cutoff value of the continuous variables for diagnosing MVI, and then, the continuous variables were converted to categorical variables for analysis. Additionally, the following qualitative imaging parameters were evaluated on a plain MR scan: (I) contour; (II) hemorrhage; (III) hepatic capsular retraction; and (IV) peritumoral bile duct dilation. Dynamic enhancement characteristics were as follows: (I) arterial enhancement pattern; (II) washout; (III) peritumoral enhancement pattern, which was defined as the enhancing portion surrounding the tumor border (15); (IV) false capsule; and (V) delayed central enhancement. Other imaging features were as follows: (VI) targetoid diffusion restriction; (VII) corona enhancement; (VIII) nodule in nodule architecture; (IX) mosaic architecture; and (X) diffusion restriction. Reviewers assigned a LI-RADS version 2018 (LI-RADS v2018) category for each hepatic observation in the study population, namely, LR-TIV (tumor in vein), LR-M (definitely or probably malignant, not HCC-specific) or LR 1-5 (1, definitely benign; 2, probably benign; 3 , indeterminate probability of HCC; 4, probably HCC; or 5 , definitely HCC) $(16,17)$. Two experienced radiologists independently reviewed the MR images. Both radiologists were aware that all patients had a high risk of liver primary cancer but were blinded to the specific diagnosis of each tumor. A consensus was reached after discussion in case of any discrepancies. Only the largest pathologically confirmed CHCC-CCA was evaluated. All surgical specimens were examined histopathologically to identify the presence of MVI, satellite nodules and LNM. MVI was defined as the presence of a tumor in the portal vein, hepatic vein, or large capsular vessel of the surrounding hepatic tissue lined by endothelium that was visible only by microscopy. Satellite nodules were defined as detached micrometastatic nodules of HCC cells embedded in the hepatic parenchyma. The pathological LNM status of each patient was identified according to the histopathological reports.

\section{Development of the combination nomograms}

Univariate and multivariate logistic regression analyses were performed to evaluate the independent clinical risk factors for the presence of MVI and satellite nodules in the training set. Considering multicollinearity among the imaging variables, the least absolute shrinkage and selection operator (LASSO) logistic regression algorithm was used to select the most useful predictive imaging features. With regard to the small number of events relative to the number of clinical variables for predicting LNM, LASSO regression was used to select both the clinical and imaging predictors of LNM. Using the coefficients derived from the LASSO logistic regression models, we then constructed a formula to calculate the risk score for each patient. Formula $=$ expression index $1 \times$ $\beta$ index $1+\ldots+$ expression index $\mathrm{n} \times \beta$ index $\mathrm{n}$ (where $\beta$ is the regression coefficient derived from LASSO regression) $(18,19)$. Independent risk clinical factors and imaging risk scores from the LASSO regression model were integrated into the nomogram for predicting MVI and satellite nodules. In addition, both the clinical risk scores and imaging risk scores obtained from the LASSO regression models were used to construct a nomogram for predicting LNM. The clinical model and imaging model were established separately using the independent clinical risk factors and the imaging risk scores obtained from the LASSO regression.

\section{Evaluation and validation of the combination nomograms and their prognostic value}

The performance of the proposed nomograms was evaluated in terms of three aspects: discrimination ability, calibration, and clinical utility. ROC curves were plotted, followed by Harrell's C-index (20) and calibration curves, with the goal of validating the discrimination ability and accuracy of the constructed nomograms in both the training and validation sets $(21,22)$. Decision curve analysis (DCA) assigns a weight to the relative value of the benefit to harm, thereby modeling the consequences of clinical decisions (23). The decision curve was plotted at different threshold probabilities for the overall patients. Kaplan-Meier curves and log rank tests were 
conducted to determine the recurrence-free survival (RFS) and overall survival (OS) differences between different risk groups based on the median risk score, which was calculated by the formulas constructed by the predicted nomograms of the MVI and satellite nodules. Time-dependent ROC curves were displayed to evaluate the performances of the predicted nomograms for the MVI and satellite nodules.

\section{Statistical analysis}

All statistical analyses were performed using R software (version 3.5.2; https://www.r-project.org/). Student's $t$ test was used to compare continuous variables with a normal distribution, and the Mann-Whitney $U$ test was used to analyze variables with an abnormal distribution. Categorical variables were compared using the chi-square test or Fisher's exact test. The reported statistical significance levels were all two-sided, and $\mathrm{P}<0.05$ was considered significant.

\section{Results}

\section{Study flowchart and clinicopathological characteristics}

In total, 171 consecutive patients with pathologically confirmed CHCC-CCA after hepatectomy between January 2015 and December 2019 who met the inclusion criteria were included. Then, 116 and 55 patients were assigned to the training set and validation set, respectively (Figure 1A,1B).

The preoperative clinicopathologic and $M R$ imaging characteristics of the patients are listed in Table 1 and Table 2. Representative CHCC-CCA images assigned as different LI-RADS categories are shown in Figure 2 and Figure 3. There was no significant difference in terms of the clinicopathologic and imaging characteristics between the training and validation sets. Histopathologically identified MVI was observed in 48 (41.4\%) and 27 (49.1\%) patients in the two sets, respectively, and satellite nodules were observed in $21(18.1 \%)$ and $9(16.4 \%)$ patients in the two sets, whereas LNM was observed in $6(5.2 \%)$ and $5(9.1 \%)$ patients in the two sets, respectively. No statistically significant difference existed in the MVI rate $(\mathrm{P}=0.342)$, satellite nodule rate $(\mathrm{P}=0.780)$ or $\mathrm{LNM}$ rate $(\mathrm{P}=0.329)$ between the two sets, verifying their use as training and validation sets.

\section{Independent prognostic factors of $M V I$, satellite nodules and LNM and nomogram construction}

Univariate analysis revealed that neutrophil to lymphocyte ratio (NLR) $(\mathrm{P}=0.049)$, platelet to lymphocyte ratio (PLR) $(\mathrm{P}=0.001)$, lymphocyte to monocyte ratio (LMR) $(\mathrm{P}=0.047)$, albumin-to-alkaline phosphatase ratio (AAPR) $(\mathrm{P}=0.008)$, tumor size $(\mathrm{P}=0.006)$ and AFP level $(\mathrm{P}=0.01)$ were associated with the presence of MVI, and tumor size $(\mathrm{P}<0.001)$ and carcinoembryonic antigen $(\mathrm{CEA})(\mathrm{P}=0.045)$ were associated with the presence of satellite nodules. In the multivariate analysis, PLR (OR, 4.8; 95\% CI: 1.1-19.7; $\mathrm{P}=0.031$ ), AAPR (OR, 4.8; 95\% CI: 1.3-17.3; $\mathrm{P}=0.017$ ) were identified as independent predictors of MVI (Table 3). Additionally, tumor size (OR, 4.3; 95\% CI: 1.1-16.1; $\mathrm{P}=0.003)$ and CEA (OR, 12.3; 95\% CI: 1.4-107.1; $\mathrm{P}=0.023)$ were significantly associated with the presence of satellite nodules (Table 4). We utilized the LASSO logistic regression algorithm to select candidate imaging features that were reliably associated with the presence of MVI (Figure $4 A, 4 B$ ), satellite nodules (Figure 4C,4D) and LNM (Figure S1A,S1B) in the training set. The formulas for the imaging scores in the LASSO models are listed in Table S1. Similarly, the LASSO logistic regression algorithm was also used to detect the clinical features associated with LNM in the training set (Figure S1C,S1D), and the formula for clinical scores in the LASSO model is listed in Table S1.

\section{Performance and validation of the nomograms}

According to the results of the multivariate logistic regression and LASSO regression, clinical risk factors and imaging features for the prediction of each outcome were identified, and then integrated nomograms were constructed by combining the clinical risk factors and imaging models for MVI (Figure $5 A$ ), satellite nodules (Figure 5B) and LNM (Figure S2). The C-index for the nomogram predicting MVI was 0.857 (95\% CI: $0.787-$ 0.927 ) in the training set and 0.795 (95\% CI: $0.674-0.916)$ in the validation set (Figure $6 A$ ); the $\mathrm{C}$-index for the prediction of satellite nodules was 0.919 (95\% CI: 0.858 0.980 ) in the training set and 0.883 (95\% CI: $0.740-0.906$ ) in the validation set (Figure $6 B$ ); and the $\mathrm{C}$-index for the nomogram predicting LNM was 0.872 (95\% CI: $0.711-$ 0.900 ) in the training set and 0.666 (95\% CI: 0.605-0.751) in the validation set (Figure S3). We also did subgroup analysis for validating the constructed model for classical type of CHCC-CCA, the C-index for the prediction of MVI, satellite nodules and LNM was $0.788,0.885$ and 0.952 respectively (Figure S4A-S4C). Calibration plots of the training and validation sets all graphically showed good agreement between the actual risk confirmed by 
Table 1 Comparisons of clinical characteristics of patients in the training and validation set

\begin{tabular}{|c|c|c|c|}
\hline Variable & Training set $(\mathrm{N}=116)(\%)$ & Validation set $(\mathrm{N}=55)(\%)$ & $P$ value ${ }^{\star}$ \\
\hline$<65$ & $95(81.9)$ & $46(83.6)$ & \\
\hline$\geq 65$ & $21(18.1)$ & $9(16.4)$ & \\
\hline Gender & & & 0.232 \\
\hline Female & $26(22.4)$ & $17(30.9)$ & \\
\hline Histopathological type & & & 0.378 \\
\hline Classical type & $108(93.1)$ & $38(69.1)$ & \\
\hline Typical subtype of stem cell features & $14(12.1)$ & $4(7.3)$ & \\
\hline Hepatitis B virus & & & 0.389 \\
\hline Negative & $19(16.4)$ & $12(21.8)$ & \\
\hline Positive & $97(83.6)$ & $43(78.2)$ & \\
\hline CA19-9, ng/mL & & & 0.367 \\
\hline$<37$ & $87(60.3)$ & $45(83.6)$ & \\
\hline$\geq 37$ & $28(39.7)$ & $10(16.4)$ & \\
\hline AFP, ng/mL & & & 0.781 \\
\hline$<400$ & $90(78.3)$ & $42(76.4)$ & \\
\hline Albumin, $g / L$ & & & 0.229 \\
\hline$<35$ & $3(2.6)$ & $0(0.0)$ & \\
\hline$\geq 35$ & $113(97.4)$ & $55(100.0)$ & \\
\hline ALT, U/L & & & 0.931 \\
\hline$<40$ & 83 (71.6) & 39 (70.9) & \\
\hline$\geq 40$ & $33(28.4)$ & $16(29.1)$ & \\
\hline AST, U/L & & & 0.738 \\
\hline$<40$ & 88 (75.9) & 43 (78.2) & \\
\hline$\geq 40$ & $28(24.1)$ & $12(21.8)$ & \\
\hline GGT, U/L & & & 0.390 \\
\hline$<50$ & 63 (54.3) & $26(47.3)$ & \\
\hline$\geq 50$ & $53(45.7)$ & $29(52.7)$ & \\
\hline
\end{tabular}

Table 1 (continued) 
Table 1 (continued)

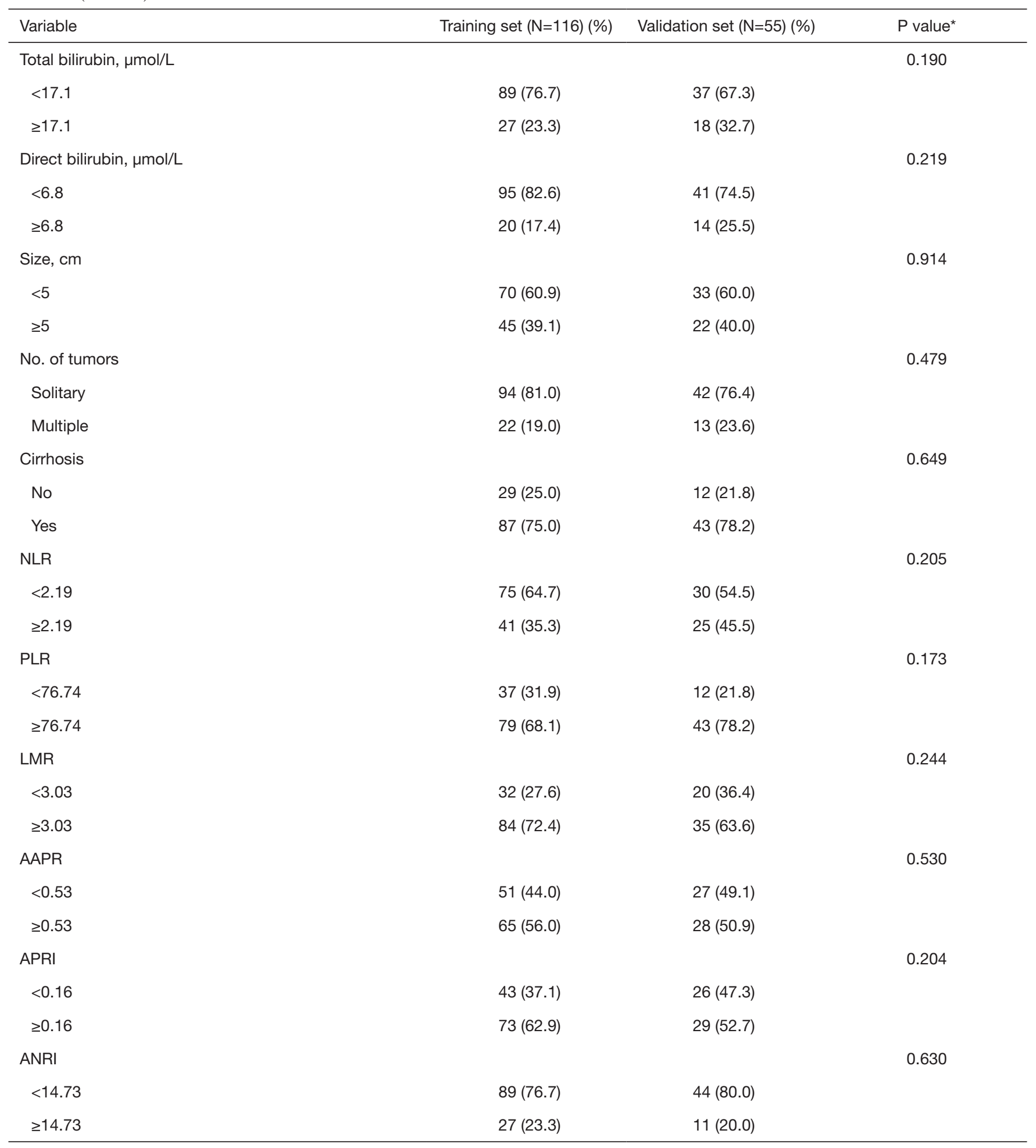

Table 1 (continued) 
Table 1 (continued)

\begin{tabular}{|c|c|c|c|}
\hline Variable & Training set $(\mathrm{N}=116)(\%)$ & Validation set $(\mathrm{N}=55)(\%)$ & $P$ value* \\
\hline Absent & $95(81.9)$ & $46(83.6)$ & \\
\hline Present & $21(18.1)$ & $9(16.4)$ & \\
\hline MVI & & & 0.342 \\
\hline Present & $48(41.4)$ & $27(49.1)$ & \\
\hline LNM & & & 0.329 \\
\hline Absent & $110(94.8)$ & $50(90.9)$ & \\
\hline Present & $6(5.2)$ & $5(9.1)$ & \\
\hline Complete & $23(19.8)$ & $9(16.4)$ & \\
\hline Incomplete & $24(20.7)$ & $11(20.0)$ & \\
\hline Tumor location & & & 0.492 \\
\hline Right liver lobe & $29(25.0)$ & $11(20.0)$ & \\
\hline Left liver lobe & $84(72.4)$ & $44(80.0)$ & \\
\hline Other location & $3(2.6)$ & $0(0.0)$ & \\
\hline
\end{tabular}

Data are shown as number of patients, with the percentage in parentheses. *, $\mathrm{P}$ value was calculated by $\chi^{2}$ test or Fisher's exact test. CA 19-9, carbohydrate antigen 19-9; AFP, $\alpha$-fetoprotein; CEA, carcinoembryonic antigen; ALT, alanine aminotransferase; AST, aspartate aminotransferase; GGT, $\gamma$-glutamyltranspeptidase; NLR, neutrophil to lymphocyte ratio; PLR, platelet to lymphocyte ratio; LMR, lymphocyte to monocyte ratio; AAPR, albumin-to-alkaline phosphatase ratio; APRI, aspartate aminotransferase to platelet ratio index; ANRI, aspartate aminotransferase to neutrophil ratio index; MVI, microvascular invasion; LNM, lymph node metastasis.

Table 2 Comparisons of MR imaging features of patients in the training and validation set

\begin{tabular}{|c|c|c|c|}
\hline Variable & Training set $(\mathrm{N}=116)(\%)$ & Validation set $(\mathrm{N}=55)(\%)$ & $P$ value ${ }^{\star}$ \\
\hline Shape & & & 0.888 \\
\hline Globular & $44(37.9)$ & $23(41.8)$ & \\
\hline Irregular & $16(13.8)$ & $7(12.7)$ & \\
\hline Contour & & & 0.888 \\
\hline Smooth & $43(37.1)$ & $21(38.2)$ & \\
\hline Hemorrhage & & & 0.766 \\
\hline Absent & 95 (81.9) & $44(80.0)$ & \\
\hline Present & $21(18.1)$ & $11(20.0)$ & \\
\hline Targetoid restriction & & & 0.413 \\
\hline
\end{tabular}

Table 2 (continued) 
Table 2 (continued)

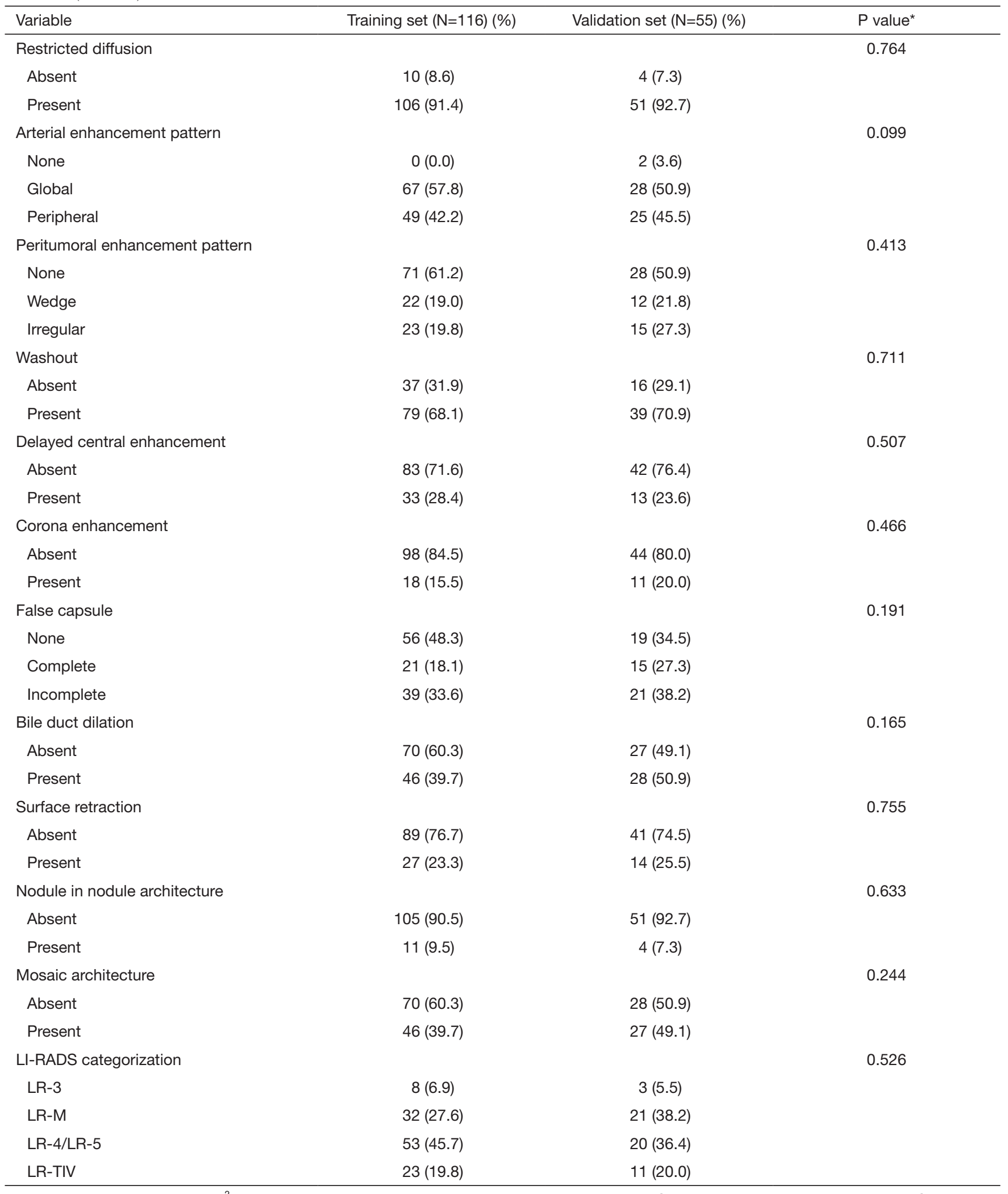

*, P value was calculated by $\chi^{2}$ test or Fisher's exact test. MR, magnetic resonance; LI-RADS, Liver Imaging Reporting and Data System. 

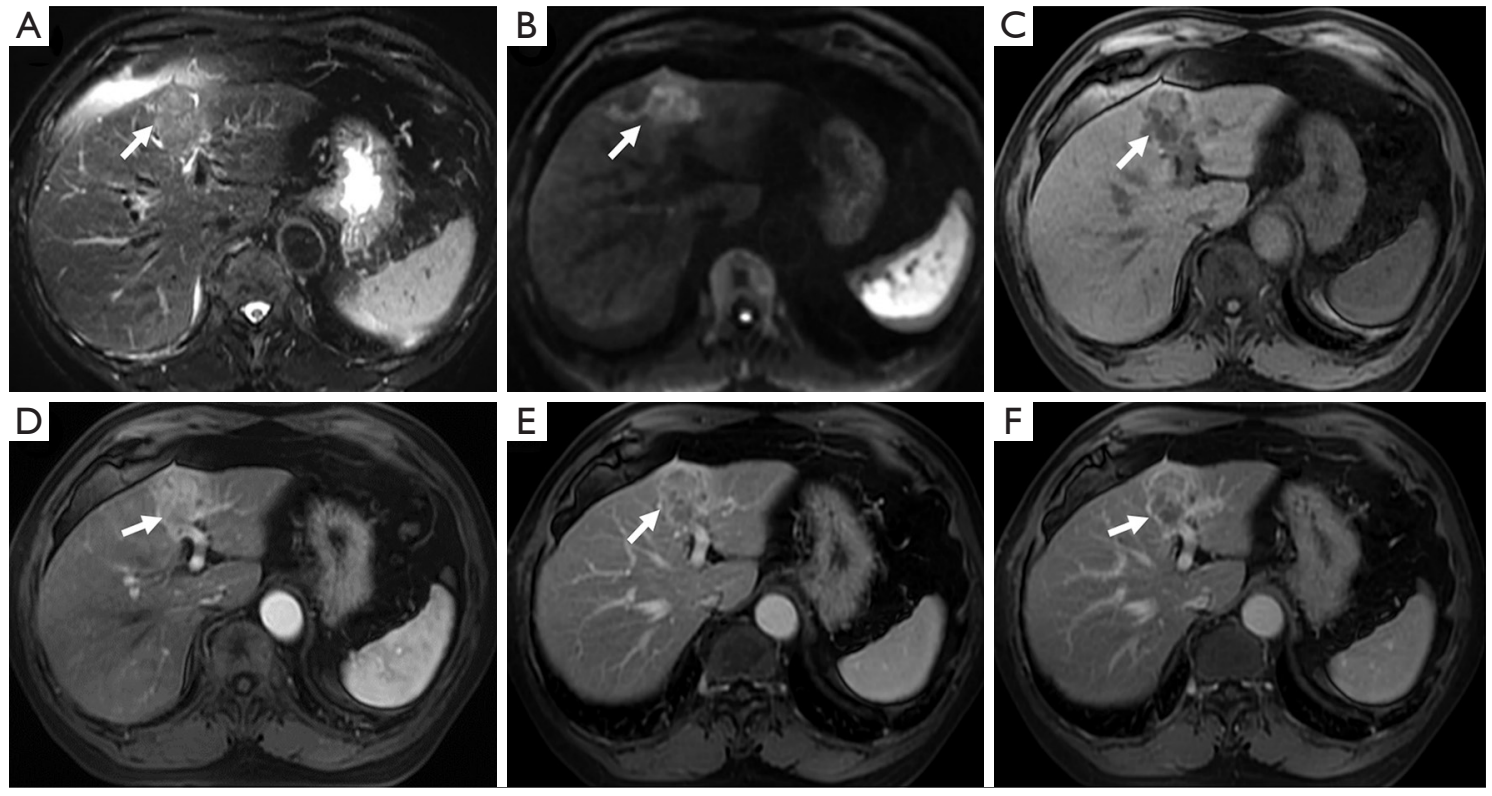

Figure 2 Images of a 66-year-old male patient with CHCC-CCA. There was an irregular tumor in left lobe of liver showing mild hyperintensity on T2-weighted imaging (A) and hyperintensity diffusion-weighted imaging (B). T1-weighted imaging (C) showed heterogeneous hypointensity. It presented non-rim arterial phase hyperenhancement (D), nonperipheral washout appearance on portal venous phase (E), and enhancing capsule (arrow) on delayed phase (F). CHCC-CCA, combined hepatocellular cholangiocarcinoma.
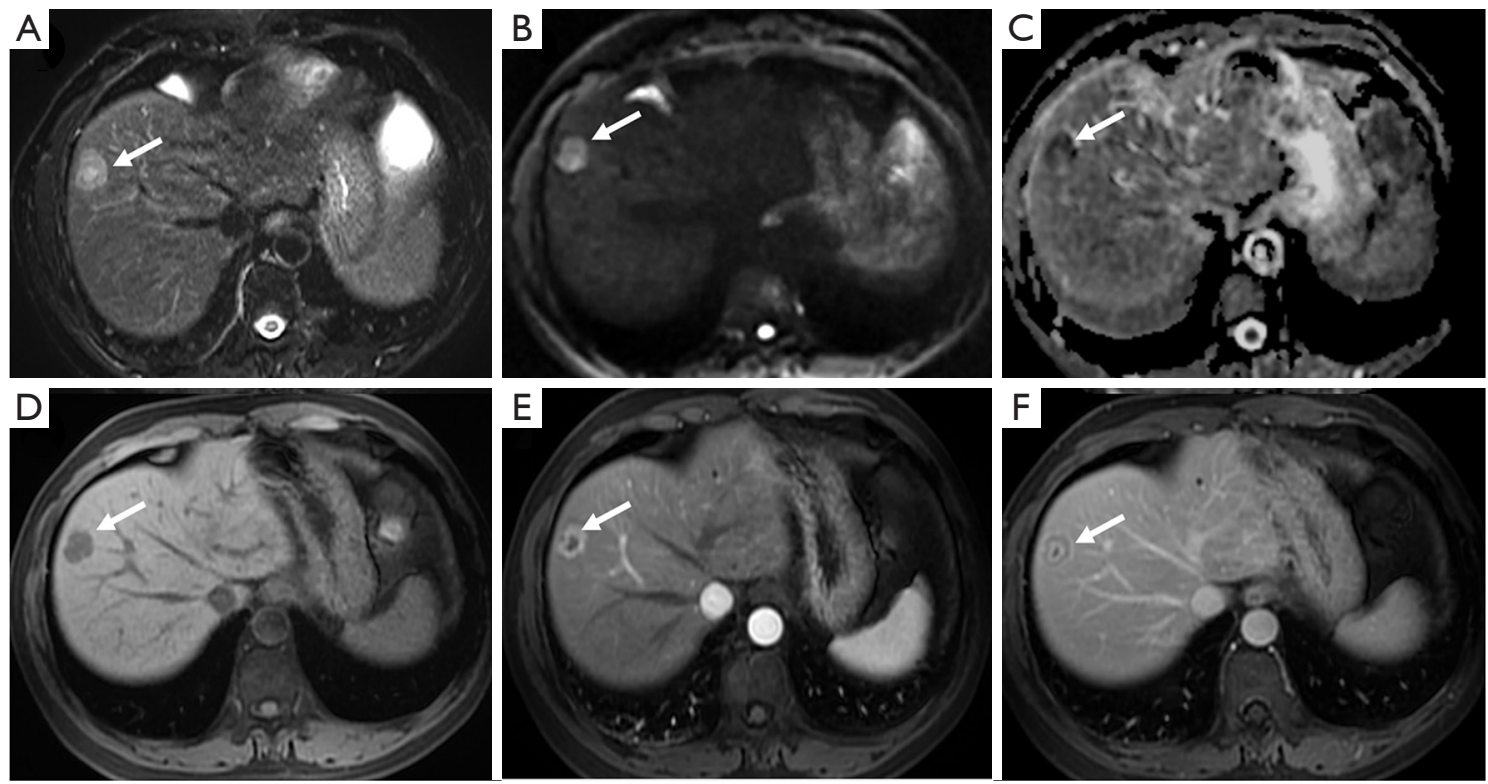

Figure 3 Images of a 47-year-old male patient with CHCC-CCA. There is a ground tumor (diameter: $2.0 \mathrm{~cm}$ ) in right lobe of liver showing heterogeneous hyperintensity on T2-weighted imaging (A). It presents hyperintensity on diffusion-weighted imaging (B) and hypointensity on ADC (C). T1-weighted imaging (D) shows homogeneous hypointensity of the lesion. Rim arterial phase hyperenhancement on contrastenhanced T1-weighted imaging (E) and peripheral washout appearance on portal venous phase (F) is presented on this lesion. CHCC-CCA, combined hepatocellular cholangiocarcinoma; ADC, apparent diffusion coefficient. 
Table 3 Univariate and multivariate analysis of MVI presence based on clinical features in the training set

\begin{tabular}{|c|c|c|c|c|c|c|}
\hline Risk factor & \multicolumn{3}{|c|}{ Univariate analysis } & \multicolumn{3}{|c|}{ Multivariate analysis } \\
\hline \multicolumn{7}{|l|}{ Age, year } \\
\hline$<65$ & 1.0 & & & & & \\
\hline$\geq 65$ & 1.1 & $0.4-2.8$ & 0.879 & & & \\
\hline Male & 1.0 & & & & & \\
\hline Female & 1.3 & $0.7-2.6$ & 0.448 & & & \\
\hline \multicolumn{7}{|l|}{ Hepatitis B virus } \\
\hline Negative & 1.0 & & & & & \\
\hline Right liver lobe & 1.0 & & & & & \\
\hline Left liver lobe & 1.5 & $0.6-3.6$ & 0.369 & & & \\
\hline Other location & 1.0 & $0.1-11.8$ & 0.968 & & & \\
\hline \multicolumn{7}{|l|}{ No. of tumors } \\
\hline Solitary & 1.0 & & & & & \\
\hline Multiple & 1.1 & $0.5-2.3$ & 0.804 & & & \\
\hline \multicolumn{7}{|l|}{ Size, cm } \\
\hline$<5$ & 1.0 & & & 1.0 & & \\
\hline$<5$ & 1.0 & & & & & \\
\hline$\geq 5$ & 1.4 & $0.5-3.6$ & 0.488 & & & \\
\hline \multicolumn{7}{|l|}{ CA19-9, ng/mL } \\
\hline$<37$ & 1.0 & & & & & \\
\hline$\geq 37$ & 0.6 & $0.2-1.5$ & 0.283 & & & \\
\hline \multicolumn{7}{|l|}{ ALT, U/L } \\
\hline$<40$ & 1.0 & & & & & \\
\hline$\geq 40$ & 0.7 & $0.3-1.7$ & 0.490 & & & \\
\hline \multicolumn{7}{|l|}{ AST, U/L } \\
\hline$<40$ & 1.0 & & & & & \\
\hline$\geq 40$ & 1.3 & $0.6-3.1$ & 0.534 & & & \\
\hline
\end{tabular}

Table 3 (continued) 
Table 3 (continued)

\begin{tabular}{cccccccc}
\hline & \multicolumn{3}{c}{ Univariate analysis } & & & & Multivariate analysis \\
\cline { 2 - 6 } & OR & $95 \% \mathrm{Cl}$ & P value & & OR & $95 \% \mathrm{Cl}$ & $\mathrm{P}$ value \\
\hline
\end{tabular}

GGT, U/L

\begin{tabular}{|c|c|c|}
\hline$<50$ & 1.0 & \\
\hline$\geq 50$ & 1.2 & $0.6-2.4$ \\
\hline
\end{tabular}

Total bilirubin, $\mu \mathrm{mol} / \mathrm{L}$

$<17.1 \quad 1.0$

$\geq 17.1 \quad 1.0$

$0.4-2.3 \quad 0.939$

Direct bilirubin, $\mu \mathrm{mol} / \mathrm{L}$

\begin{tabular}{|c|c|c|}
\hline$<6.8$ & 1.0 & \\
\hline$\geq 6.8$ & 0.6 & $0.2-1.6$ \\
\hline
\end{tabular}

Albumin, g/L

$$
<35
$$

$\geq 35$

NLR

$$
\begin{aligned}
& <2.19 \\
& \geq 2.19
\end{aligned}
$$

PLR

$$
\begin{aligned}
& <76.74 \\
& \geq 76.74
\end{aligned}
$$

LMR

$$
<3.03
$$$$
\geq 3.03
$$

AAPR

$<0.53$

$\geq 0.53$

APRI

$$
\begin{aligned}
& <0.16 \\
& \geq 0.16
\end{aligned}
$$

ANRI

$\begin{array}{ll}<14.73 & 1.0 \\ \geq 14.73 & 1.4\end{array}$

1.0

1.0

2.2

1.0

4.6

1.0

0.4

1.0

2.9

0.6

$\begin{array}{lll}1.4 & 0.1-16.2 & 0.775\end{array}$

$1.0-4.7$

0.049

1.0

0.9

$0.2-3.6$

0.901

$1.8-11.8$

0.001

1.0

4.8

$1.1-19.7$

0.031

$0.2-1.0$

0.047

1.0

0.3

$0.1-1.3$

0.098

1.3-6.4

0.008

1.0

4.8

1.3-17.3

0.017

$0.3-1.3$

0.212

MVI, microvascular invasion; OR, odds ratio; Cl, confidence interval; AFP, $\alpha$-fetoprotein; CEA, carcinoembryonic antigen; CA 19-9, carbohydrate antigen 19-9; ALT, alanine aminotransferase; AST, aspartate aminotransferase; GGT, $\gamma$-glutamyltranspeptidase; NLR, neutrophil to lymphocyte ratio; PLR, platelet to lymphocyte ratio; LMR, lymphocyte to monocyte ratio; AAPR, albumin-to-alkaline phosphatase ratio; APRI, aspartate aminotransferase to platelet ratio index; ANRI, aspartate aminotransferase to neutrophil ratio index. 
Table 4 Univariate and multivariate analysis of presence of satellite nodules based on clinical features in the training set

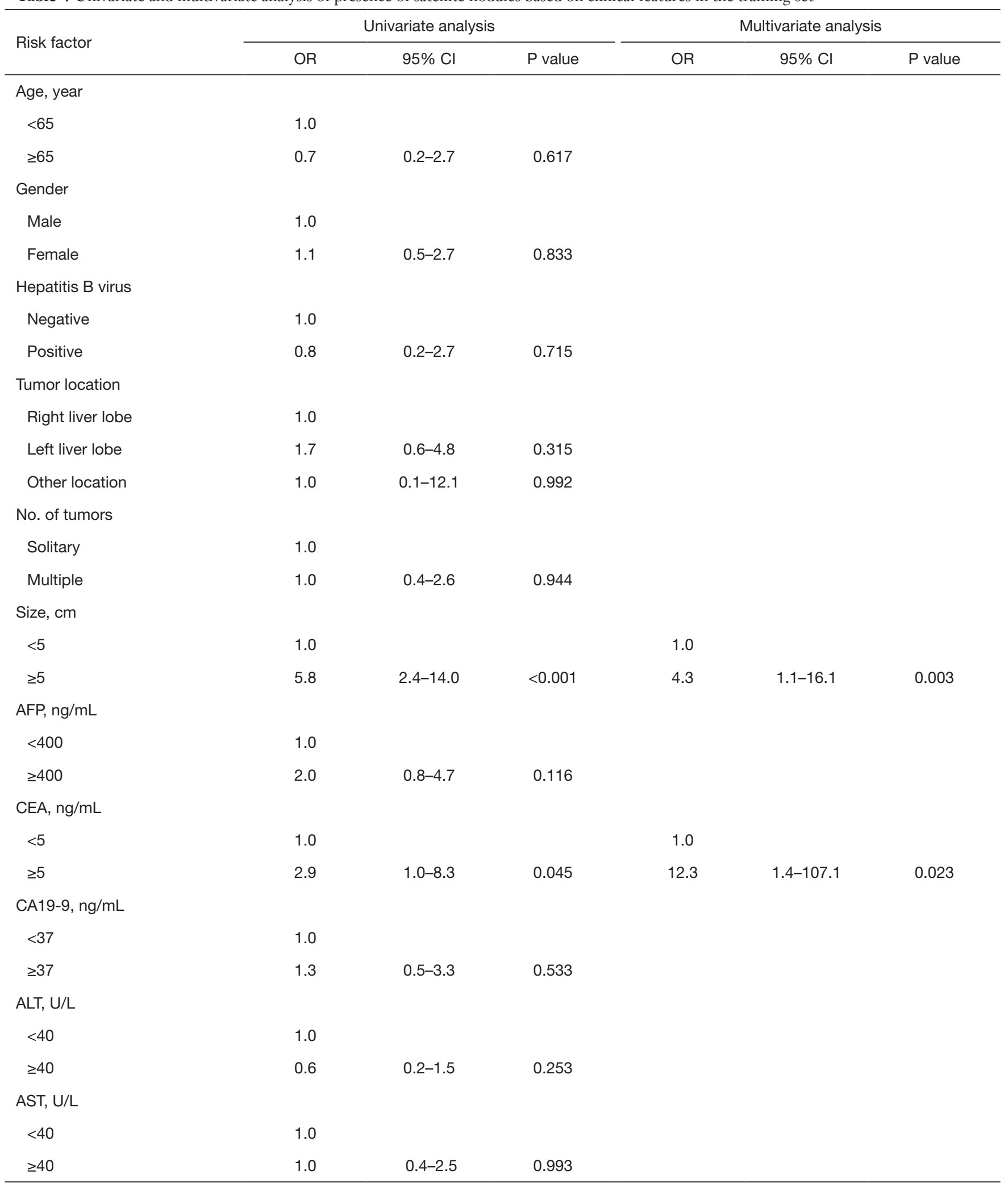

Table 4 (continued) 
A

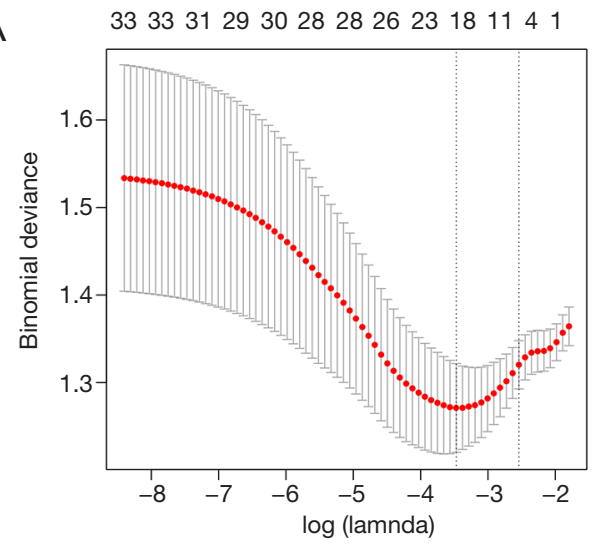

C

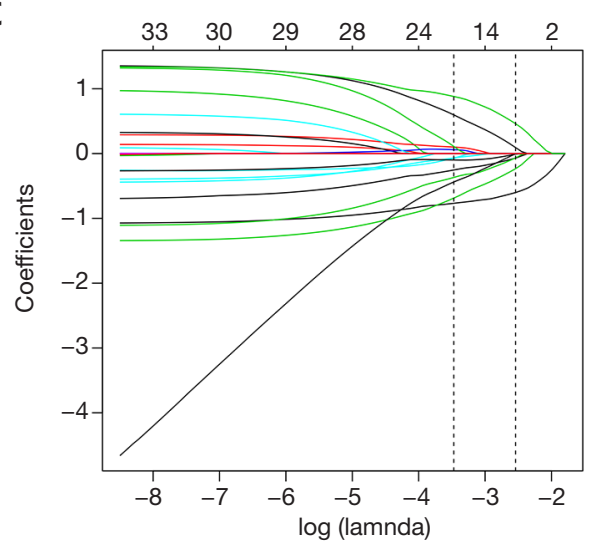

B

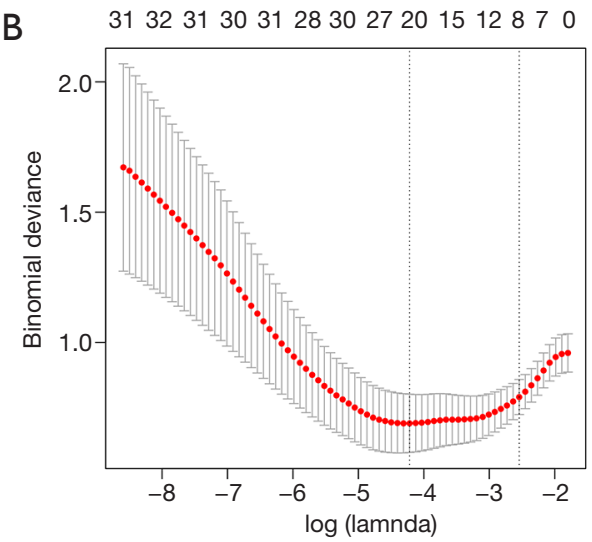

D

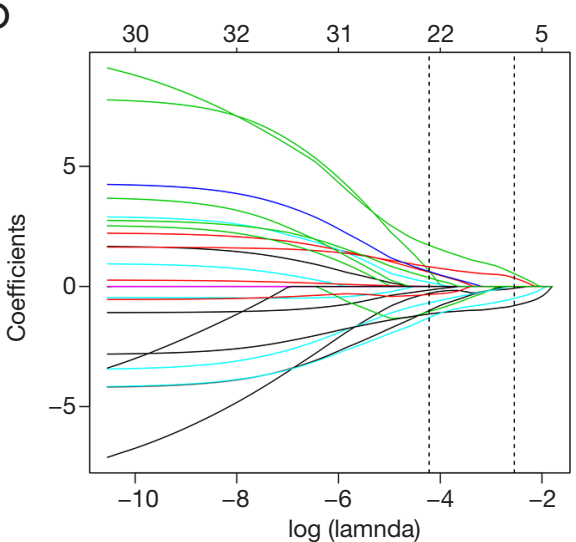

Figure 4 Selection of imaging features using the LASSO logistic regression algorithm. The penalization coefficient $\lambda$ in the LASSO model was tuned using 10-fold cross-validation and minimum criterion for presence of MVI (A) and satellite nodules (B). The lower X-axis shows $\log (\lambda)$, while the top $\mathrm{X}$-axis indicates the number of predictors for the given $\log (\lambda)$. The $\mathrm{Y}$-axis indicates binomial deviance. Red dots represent average misclassification errors for every model with given $\lambda$, and the vertical bars indicate the upper and lower values of the misclassification errors. The vertical black lines define the optimal $\lambda$, which provides its best fit to the data. As a result, an optimal $\lambda$ of 0.031 , with $\log (\lambda)=-3.471$, was selected for MVI prediction and an optimal $\lambda$ of 0.028 , with $\log (\lambda)=-3.589$, was selected for satellite nodules prediction. LASSO coefficient profiles of the imaging characteristics of MVI (C) and satellite nodules (D). MVI, microvascular invasion; LASSO, least absolute shrinkage, and selection operator.

pathological examination and the predicted risk for MVI (Figure $7 A, 7 B$ ), satellite nodules (Figure $7 C, 7 D$ ) and LNM (Figure S5) in both the training and validation sets. The decision curves of the combined nomograms, clinical model and imaging model are shown in Figure $8 \mathrm{~A}$ for MVI, Figure $8 B$ for satellite nodules and Figure S6 for LNM, indicating that our nomograms offered more benefits than the treat-all or treat-none schemes.

We observed C-indexes corresponding to the area of the ROC curves among different models for predicting MVI, satellite nodules and LNM and found that the combined nomograms were superior to those of both the clinical and imaging models for the prediction of all outcomes in the training set and validation set. According to the ROC analysis, our constructed nomograms had improved predictive value for MVI, satellite nodules and LNM and a higher threshold probability compared to the other models. The specific performances of the combined nomogram, clinical model and imaging model are summarized in Table 5.

\section{Prognostic value of the predicted MVI risk status and satellite nodule status}

To evaluate the role of the constructed nomograms in 
A

Points

AAPR

PLR

Imaging model

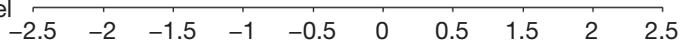

Total points

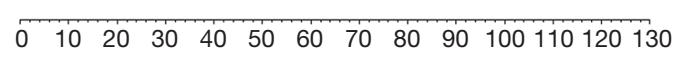

Linear predictor

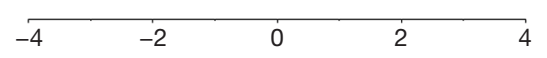

Risk of MVI

$\begin{array}{lllllllllll}0 & 10 & 20 & 30 & 40 & 50 & 60 & 70 & 80 & 90 & 100\end{array}$

$$
<0.53 \quad \geq 0.53
$$

$$
<7 \overbrace{}^{\geq 76.74}
$$

$\begin{array}{lll}0.1 & 0.5 & 0.9\end{array}$

B

Points

$\begin{array}{lllllllllll}0 & 10 & 20 & 30 & 40 & 50 & 60 & 70 & 80 & 90 & 100\end{array}$

CEA $\quad<5$

Size $\quad<5$

Imaging model

$\begin{array}{llllllllllll}-4.5 & -4 & -3.5 & -3 & -2.5 & -2 & -1.5 & -1 & -0.5 & 0 & 0.5 & 1\end{array}$

Total points

$\begin{array}{lllllllllllll}0 & 10 & 20 & 30 & 40 & 50 & 60 & 70 & 80 & 90 & 100 & 110 & 120\end{array}$

Linear predictor

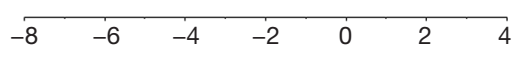

Risk of satellite nodule $\begin{array}{lll}0.1 & 0.5 & 0.9\end{array}$

Figure 5 Nomogram for predicting the presence of MVI (A) and satellite nodules (B) preoperatively. Points are assigned for PLR, AAPR and imaging model for predicting the presence of MVI. Points are assigned for CEA, size, and imaging model for predicting the presence of satellite nodules. The score for each value is assigned by drawing a line upward to the points line, and the sum of score is plotted on the Total points line. MVI, microvascular invasion; PLR, platelet to lymphocyte ratio; AAPR, albumin-to-alkaline phosphatase ratio; CEA, carcino-embryonic antigen.

A

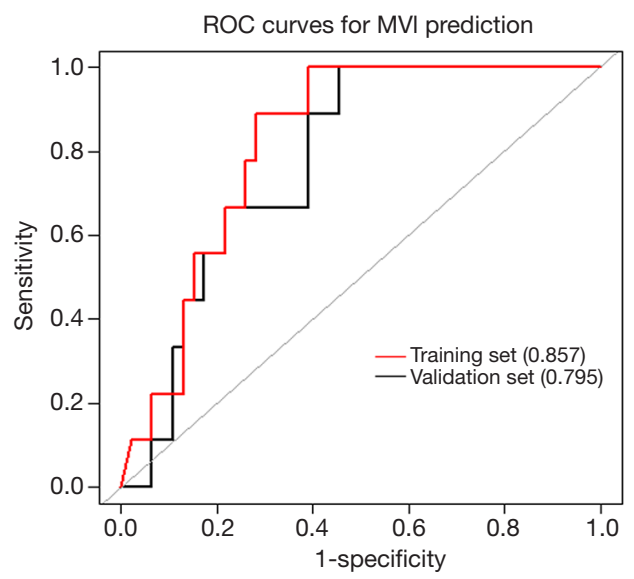

B

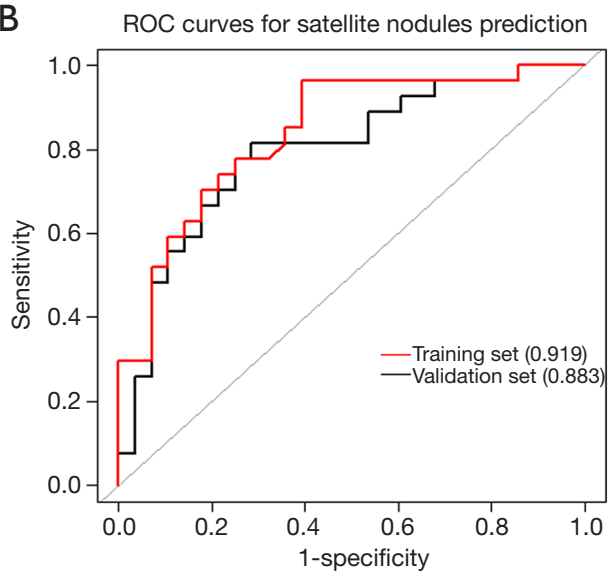

Figure 6 Receiver operating curve for prediction of MVI (A) and satellite nodules (B) using the combination nomogram in the training set and validation set. ROC, receiver operating characteristic; MVI, microvascular invasion.

the prediction of RFS and OS, we divided patients into high-risk and low-risk groups based on the median risk score calculated by the nomograms for the prediction of MVI and satellite nodules. The median risk scores for MVI and satellite nodules were -0.546 and -2.539 , respectively. Survival analysis showed that patients with a high risk of MVI $(\mathrm{P}=0.003)$ (Figure $9 A)$ and satellite nodules $(\mathrm{P}=0.042)$ (Figure 9B) had significantly worse RFS than their counterparts. Although there was no statistical difference observed in OS of different risk groups of MVI $(\mathrm{P}=0.057)$ (Figure 9C), patients with a high risk of having satellite nodules presented with worse OS than patients from the low-risk group $(\mathrm{P}=0.006)$ (Figure 9D). Furthermore, the performances of the predicted risk status of presenting MVI and satellite nodules for the prediction of OS at 1, 2 and 3 years were $0.755,0.659$ and 0.587 , and $0.769,0.722$ and 0.681 , respectively (Figure 9E,9F). 
A

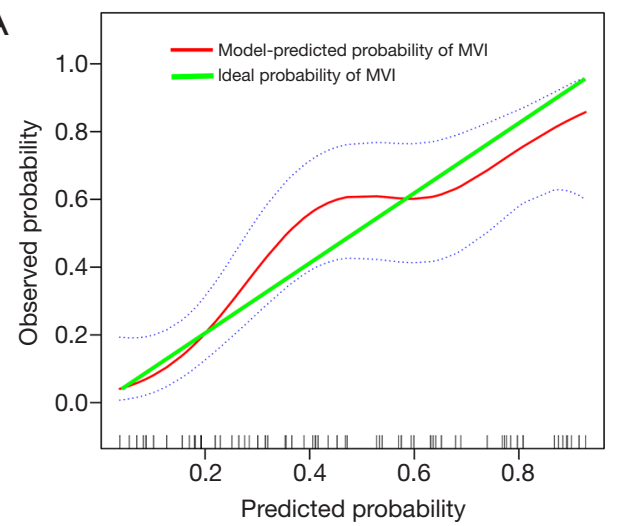

C

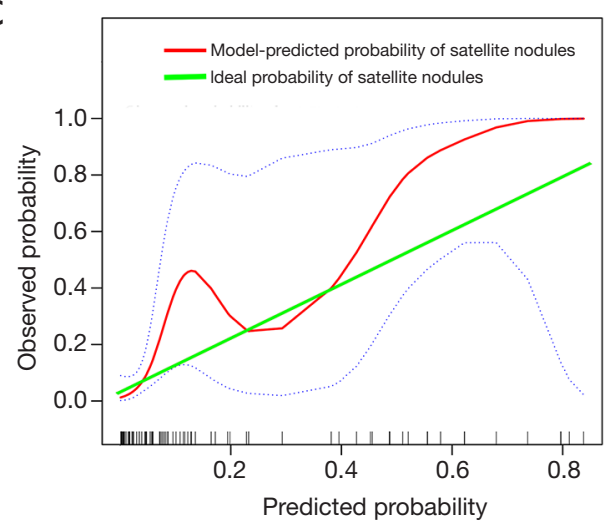

B

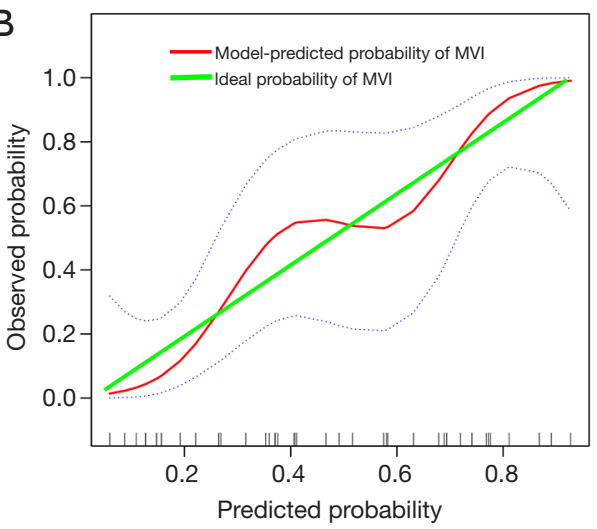

D

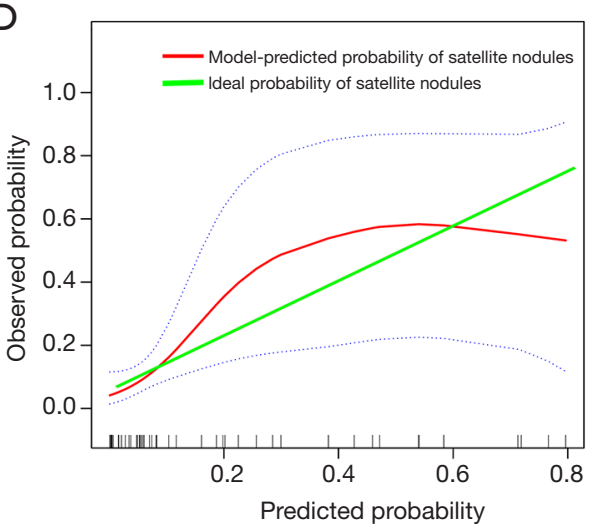

Figure 7 The calibration curves of the combination nomogram for prediction of MVI and satellite nodules in the training set $(A, C)$ and the validation set (B,D). Vertical axis: the observed probability of MVI and satellite nodules probabilities; horizontal axis: the nomogram predicted MVI and satellite nodules probabilities. MVI, microvascular invasion.

\section{Discussion}

CHCC-CCA has several overlapping imaging features with ICC and HCC because of hepatocellular or cholangiocellular differentiation in variable proportion, which could lead to misdiagnosis before surgery. As a result, the misdiagnosis could not guarantee the optimal treatment choice for patients with CHCC-CCA. Recently, abundant researches have proposed diagnostic algorithm of CHCCCCA. Gigante et al. (24) developed a two-step strategy combining imaging and tumor biopsy, achieving satisfactory diagnostic performance. Moreover, according to the LIRADS (25), tumor with imaging features of malignancy but are not specific for HCC are categorized as LR-M, and of definitive features for HCC are categorized as LR$4 / 5$, which means that if CHCC-CCA could be categorized as LR-M, it can be correctly diagnosed with the help of biopsy before surgery. It was reported that CHCC-CCAs were categorized as LR-M and LR-5/4 in $61.4 \%$ and $37.1 \%$ respectively (8). Using LI-RADS, a substantial proportion of CHCC-CCAs can be categorized as LR-M. In this scenario, given that advances in the preoperative diagnosis of CHCCCCA could be conveniently applied in the clinical cases, our nomograms for predicting MVI, satellite nodules and LNM preoperatively could further facilitate treatment strategy optimization for a subset of patients who can have definitive diagnosis using strategy of combined imaging and biopsy, or patients who are categorized as LR-M.

The intrinsic properties of CHCC-CCA differ from those of HCC and ICC. Moreover, its OS rate has been reported to be even lower than that of ICC (26-28). As a result, it is critical to separately map prediction models for strong prognostic parameters, including MVI, satellite nodules and LNM. Our results demonstrated that the combined nomogram models constructed for MVI, satellite nodules and LNM had satisfactory prognostication performance in both the training and validation sets and showed generally higher predictive value than the clinical 
A

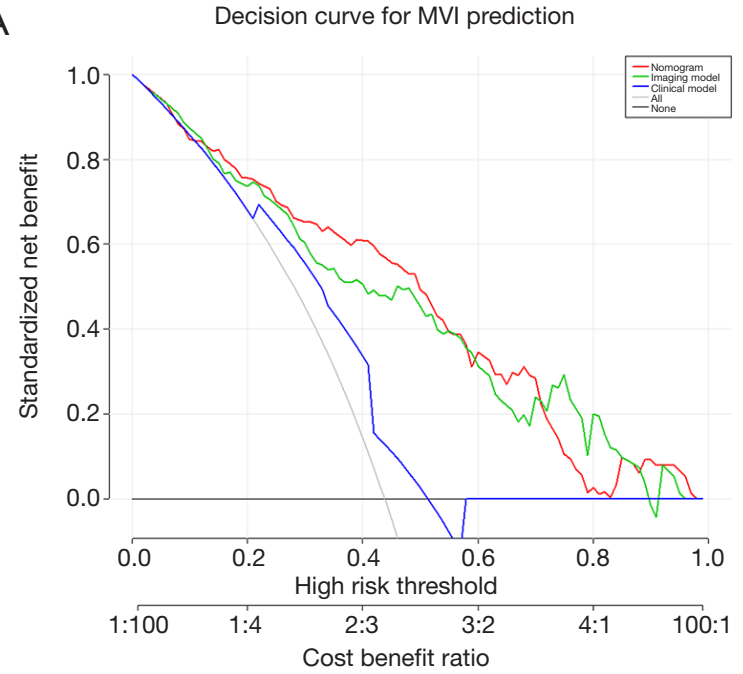

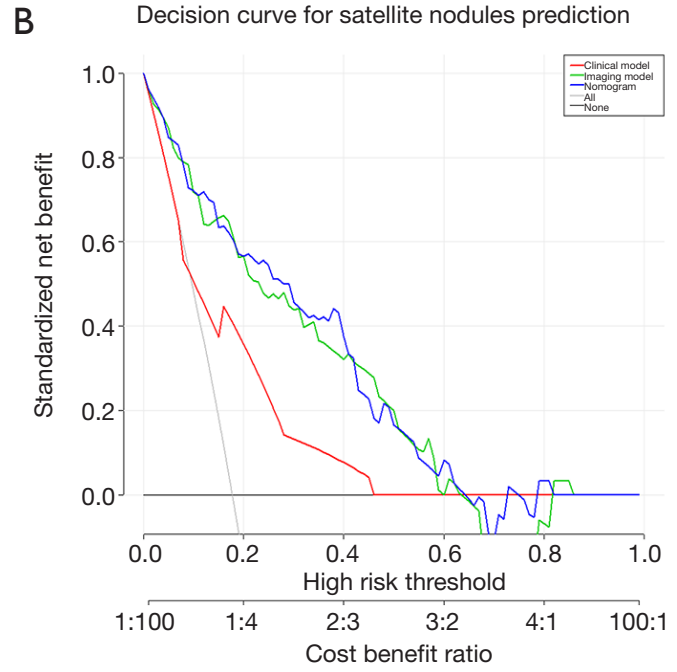

Figure 8 The decision curves of the combination nomogram, clinical model, and imaging model for prediction of MVI (A) and satellite nodules (B) in overall patients. Vertical axis: the net benefit; horizontal axis: the threshold probability at a range of 0.0 to 1.0 . The gray line represents the decision curve of the assumption that all patients suffer from MVI or satellite nodules; the black line represents the decision curve of the assumption that no patients suffer from MVI or satellite nodules. MVI, microvascular invasion.

and imaging models.

MVI is defined as the presence of tumor cells within a vascular lumen that is visible only by microscopy $(29,30)$. In contrast with MVI, satellite nodules are separate micrometastatic lesions considered to be direct malignant cell invasion of the hepatic parenchyma (31). Activation of epithelial-mesenchymal transition (EMT) transcriptional programs has been proven to be an essential pathogenic step leading to the development of MVI and satellitosis (32). Since both are associated with aggressive tumor behavior, they probably have overlapping prognostic outcomes to some extent, which can be inferred from the multiple studies in which MVI was reported to be an independent risk factor for recurrence, similar to satellite nodules $(33,34)$. Although there has been no precise classification system for MVI burden, several studies have identified the necessity of proposing novel stage criteria, as they found that the severity of MVI was highly associated with different prognoses $(35,36)$. Therefore, adverse factors for predicting different degrees of MVI are of great importance and should be analyzed further. LNM is another important predictor of a poor prognosis in CHCC-CCA patients. Hilar LNM is rarely observed in HCC patients but is often observed in patients with CHCC-CCA and ICC (10). An accurate understanding of lymph node status enables surgeons to better tailor treatment. Although our established combined nomogram showed high C-indexes for LNM prediction in both the training and validation sets, these findings should be further validated in larger cohorts.

The proposed nomograms integrated clinical and imaging risk factors and were demonstrated to be more reliable for the prediction of MVI, satellite nodules and LNM than the nomograms constructed from clinical factors or imaging factors alone. A recent study integrated clinical risk factors and laboratory blood indicators to investigate the diagnostic accuracy of MVI and satellite nodules for CHCC-CCA, yielding C-indexes of 0.826 and 0.771 , respectively (37), which were lower than those of the nomograms we built. In another study, imaging features of intratumoral fat deposition and irregular peritumoral enhancement combined with the AFP level for the prediction of MVI yielded a specificity of $98.2 \%$ but a sensitivity of $12.5 \%$ (38).

The inflammatory response plays a decisive role in cancer initiation, malignant transformation and MVI by promoting epigenetic changes, cell proliferation, angiogenesis, and cell invasion (39). In our study, the level of PLR was significant serum inflammatory markers of MVI in CHCC-CCA, which was consistent with prior studies. A meta-analysis showed that elevated PLR suggested poor OS in HCC, with a pooled HR of 1.63 (40). Albumin is also a useful marker for determining the inflammatory response, and alkaline 
Table 5 Performances of the clinical model, imaging model and combination nomogram

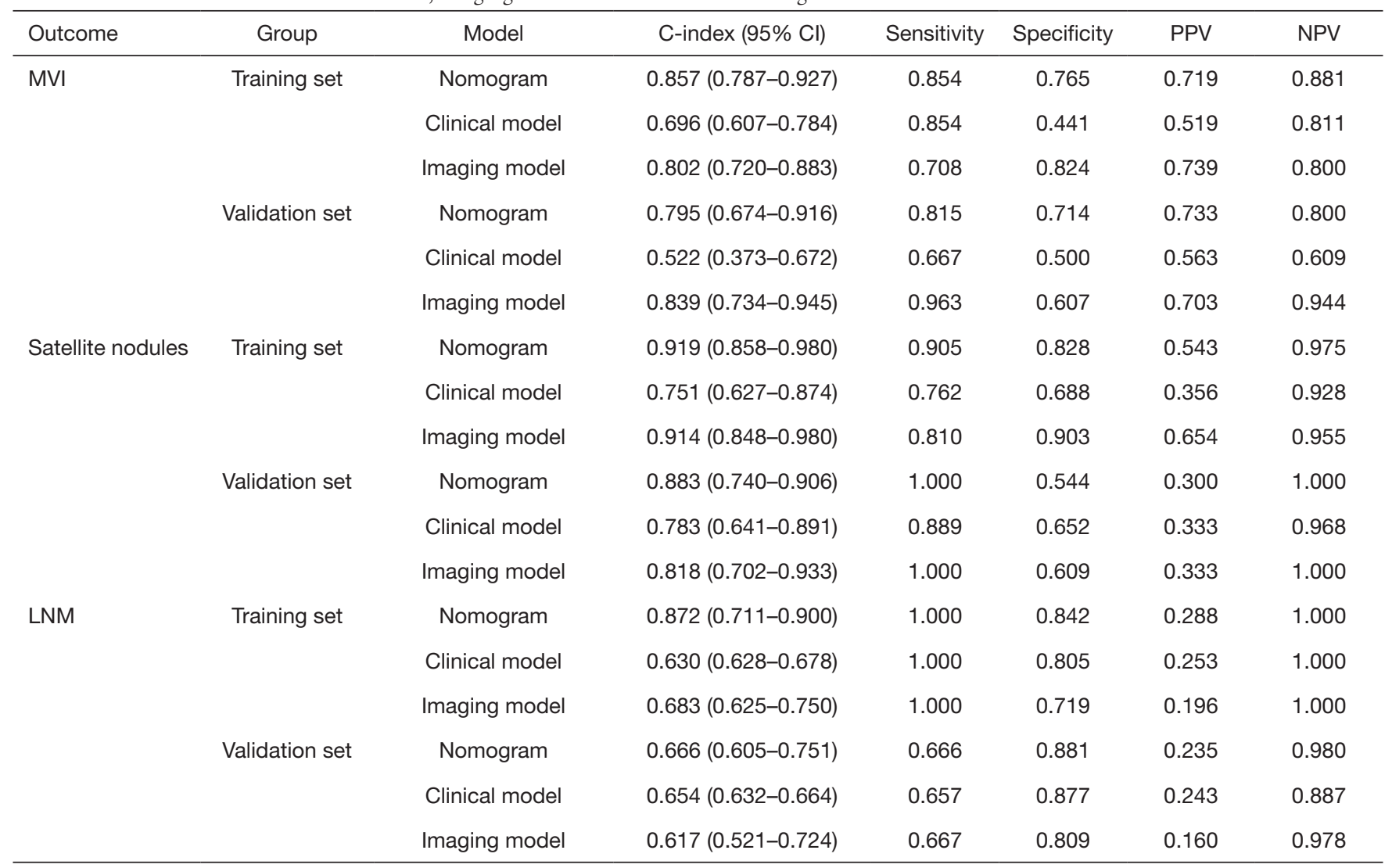

$\mathrm{CI}$, confidence interval; PPV, positive predictive value; NPV, negative predictive value; MVI, microvascular invasion; LNM, lymph node metastasis.

phosphatase hydrolase enzymes are primarily found in the liver, bile duct and bone. In our nomogram, AAPR was an independent predictor of MVI, concordant with the findings reported in the study by Zhang et al. (41), who identified that AAPR was a strong indicator of OS in CHCC-CCA, with an optimal threshold value of 0.43 . Similarly, utilizing these inflammatory indexes in our nomogram could improve its predictive power. Interestingly, PLR and AAPR were not independent risk factors for the prediction of satellite nodules in our study, probably because of the smaller number of events in satellite nodules compared with that in MVI, which led to weakened statistical power.

Imaging can help to visualize the entire profile of the relative proportions of tumor features. Several studies have reported that lesions harboring a hypervascular pattern had a larger proportion of HCC features and less fibrotic stroma $(42,43)$, while lesions with a targeted appearance, as defined by LI-RADS, were more likely to have a larger proportion of CC features and fibrotic stroma (38). The intrinsic heterogeneity of CHCC-CCA histology contributes to the discordance in survival outcomes. This idea was based on the evidence reported by Park et al. (42), demonstrating that CHCC-CCA patients with hypervascular features had better survival outcomes than CHCC-CCA patients with nonhypervascular features; simultaneously, patients categorized as having LR-M or LR-4/5 were found to have significant differences in survival outcomes $(7,8)$. The multiple imaging biomarkers selected by LASSO logistic regression in our study yielded satisfactory prediction performance. However, interobserver variability hampers the quality of imaging feature evaluations, and radiomics has begun to emerge as a useful tool to predict the status of MVI and LNM in HCC $(44,45)$; however, such a method has not yet been applied to CHCC-CCA. In the future, the establishment of the genomic, transcriptomic, proteomic and radiomic landscapes of CHCC-CCA is desirable for a better understanding of the progression of CHCC-CCA and adverse prognostic factors. 
A
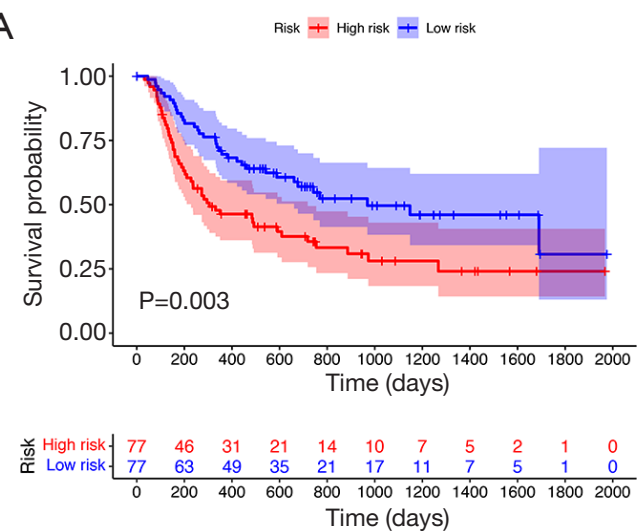

C

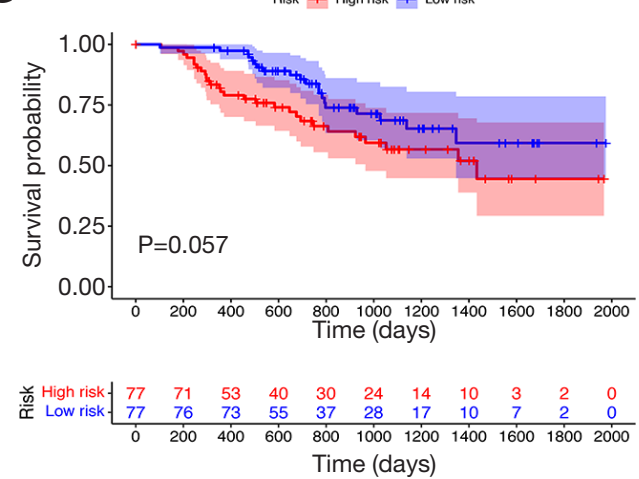

E

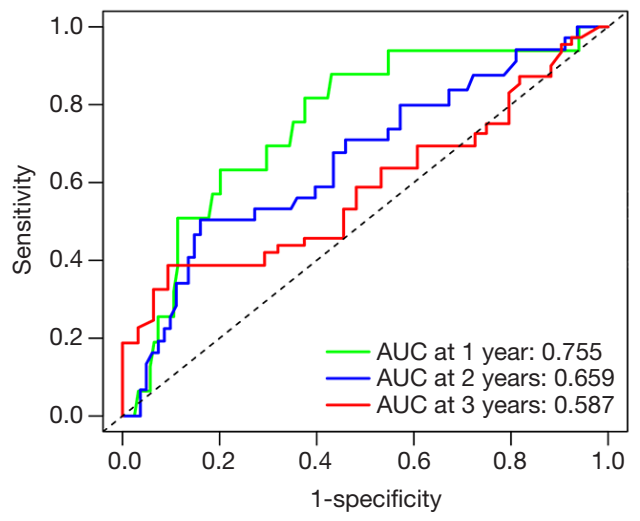

B $\quad$ Risk + Highrisk + Low risk
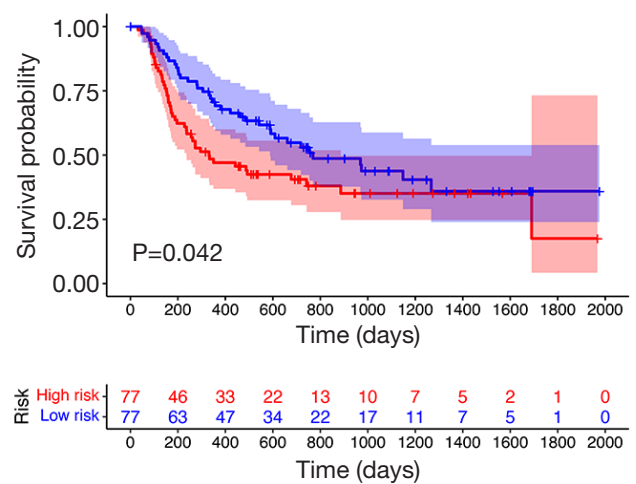

D Risk + Highrisk + Low risk
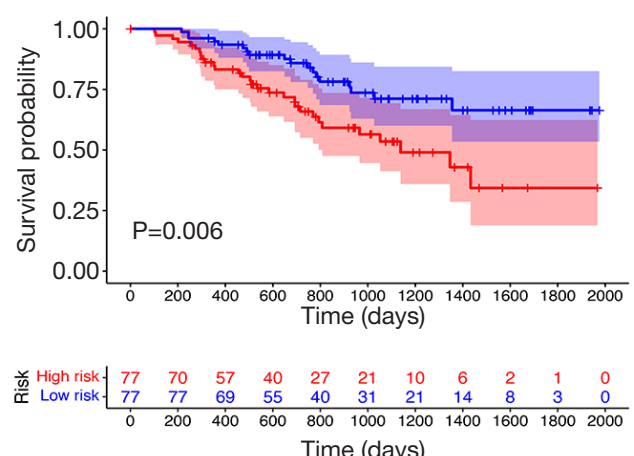

$\mathrm{F}$

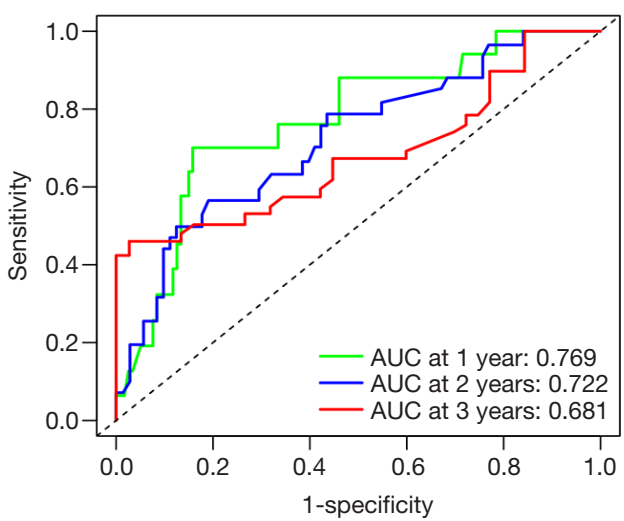

Figure 9 The prognostic value of the combination nomogram of MVI and satellite nodules stratified by different risk groups. Recurrence free survival curves were drawn by the predicted risk status of MVI (A) and satellite nodules (B) by the combination nomogram. OS curves were drawn by the predicted risk status of MVI (C) and satellite nodules (D). ROC curves for predicted risk status of MVI (E) and satellite nodules (F) for predicting OS in the total cohort. MVI, microvascular invasion; ROC, receiver operating characteristic; OS, overall survival.

Unfortunately, there is no globally accepted standard first-line therapy for CHCC-CCA, and comparative data on different therapeutic tools are sparse. Thus, the nomograms we constructed for predicting the most predominant prognostic factors might serve as an effective tool to guide appropriate clinical treatment selection. Evidence from a multicenter analysis showed that compared with resection, the outcomes of patients with CHCC-CCA who underwent transplantation were superior (46); however, they exhibited inferior 5-year survival outcomes compared to those with HCC who underwent transplantation (47). Moreover, patients with a high MVI status were reported 
to be unsuitable for transplantation since unsatisfactory OS outcomes were observed compared with patients without MVI (48). Therefore, preoperative estimated models could facilitate clinicians in recruiting candidates who could obtain the most benefit from transplantation. For patients who present with MVI or satellitosis, a larger resection margin could potentially clear intrahepatic metastases. In this scenario, RFA, as an alternative ablative therapy of hepatic resection, showed less favorable surgical outcomes due to inferior radical tumor clearance. Neoadjuvant therapy for ICC with LNM is expected to improve survival (49), providing supportive evidence that patients could receive neoadjuvant therapy for CHCC-CCA. There is also accumulating evidence suggesting that aggressive surgical treatment, including lymph node dissection, can prolong the survival of patients with CHCC-CCA (28). In this scenario, successful preoperative prediction of LNM risk could result in improved postoperative survival outcomes.

Our study had some limitations that should be mentioned. First, since our validation set was derived from a single institution, our available sample size was relatively small. Hence, analysis based on a larger cohort and external validation in other centers is necessary. Second, a prospective study is required to further confirm the reliability of our nomograms. Third, we did not categorize CHCC-CCA into different subtypes according to the latest WHO classification system; more specific prognostication among various types should be conducted to further elucidate the biological behavior of CHCC-CCA. Finally, since qualitative imaging features suffer from some limitations, including interobserver variability and lack of external validation, studies incorporating radiomics or genomics into predictive nomograms should be conducted in the future.

\section{Conclusions}

Nomograms incorporating clinical factors and imaging features achieved satisfactory performance in individualized prediction of preoperative status of MVI, the presence of satellite nodules and LNM, allowing treatment strategy optimization in patients with CHCC-CCA. Furthermore, these investigations need to be validated in more prospective studies.

\section{Acknowledgments}

Funding: This work was supported by grants from the
National Natural Science Foundation of China (grant number 91859107), the Shanghai Science and Technology Committee (grant number 19411965500), the Shanghai Municipal Key Clinical Specialty (shslczdzk03202), Clinical Research Plan of SHDC (SHDC2020CR1029B).

\section{Footnote}

Reporting Checklist: The authors have completed the TRIPOD reporting checklist. Available at https://dx.doi. org/10.21037/atm-21-2500

Data Sharing Statement: Available at https://dx.doi. org/10.21037/atm-21-2500

Conflicts of Interest: All authors have completed the ICMJE uniform disclosure form (available at https://dx.doi. org/10.21037/atm-21-2500). The authors have no conflicts of interest to declare.

Ethical Statement: The authors are accountable for all aspects of the work in ensuring that questions related to the accuracy or integrity of any part the work are appropriately investigated and resolved. This retrospective study was conducted in accordance with the Declaration of Helsinki (as revised in 2013). The study was approved by the Clinical Research Ethics Committee of Zhongshan hospital of Fudan University (B2019-139R) and individual consent for this retrospective analysis was waived.

Open Access Statement: This is an Open Access article distributed in accordance with the Creative Commons Attribution-NonCommercial-NoDerivs 4.0 International License (CC BY-NC-ND 4.0), which permits the noncommercial replication and distribution of the article with the strict proviso that no changes or edits are made and the original work is properly cited (including links to both the formal publication through the relevant DOI and the license). See: https://creativecommons.org/licenses/by-nc-nd/4.0/.

\section{References}

1. Ramai D, Ofosu A, Lai JK, et al. Combined Hepatocellular Cholangiocarcinoma: A Population-Based Retrospective Study. Am J Gastroenterol 2019;114:1496-501.

2. Seehawer M, D'Artista L, Zender L. The Worst from Both Worlds: cHCC-ICC. Cancer Cell 2019;35:823-4.

3. Ma X, Wei J, Gu D, et al. Preoperative radiomics 
nomogram for microvascular invasion prediction in hepatocellular carcinoma using contrast-enhanced CT. Eur Radiol 2019;29:3595-605.

4. Zhang X, Ruan S, Xiao W, et al. Contrast-enhanced CT radiomics for preoperative evaluation of microvascular invasion in hepatocellular carcinoma: A two-center study. Clin Transl Med 2020;10:e111.

5. Yan Y, Zhou Q, Zhang M, et al. Integrated Nomograms for Preoperative Prediction of Microvascular Invasion and Lymph Node Metastasis Risk in Hepatocellular Carcinoma Patients. Ann Surg Oncol 2020;27:1361-71.

6. Mitchell DG, Bruix J, Sherman M, et al. LI-RADS (Liver Imaging Reporting and Data System): summary, discussion, and consensus of the LI-RADS Management Working Group and future directions. Hepatology 2015;61:1056-65.

7. Choi SH, Lee SS, Park SH, et al. LI-RADS Classification and Prognosis of Primary Liver Cancers at Gadoxetic Acid-enhanced MRI. Radiology 2019;290:388-97.

8. Jeon SK, Joo I, Lee DH, et al. Combined hepatocellular cholangiocarcinoma: LI-RADS v2017 categorisation for differential diagnosis and prognostication on gadoxetic acid-enhanced MR imaging. Eur Radiol 2019;29:373-82.

9. Brunt E, Aishima S, Clavien PA, et al. cHCC-CCA: Consensus terminology for primary liver carcinomas with both hepatocytic and cholangiocytic differentation. Hepatology 2018;68:113-26.

10. Yin X, Zhang BH, Qiu SJ, et al. Combined hepatocellular carcinoma and cholangiocarcinoma: clinical features, treatment modalities, and prognosis. Ann Surg Oncol 2012;19:2869-76.

11. Kim KH, Lee SG, Park EH, et al. Surgical treatments and prognoses of patients with combined hepatocellular carcinoma and cholangiocarcinoma. Ann Surg Oncol 2009;16:623-9.

12. Lu XY, Xi T, Lau WY, et al. Hepatocellular carcinoma expressing cholangiocyte phenotype is a novel subtype with highly aggressive behavior. Ann Surg Oncol 2011;18:2210-7.

13. Kim TH, Kim H, Joo I, et al. Combined HepatocellularCholangiocarcinoma: Changes in the 2019 World Health Organization Histological Classification System and Potential Impact on Imaging-Based Diagnosis. Korean J Radiol 2020;21:1115-25.

14. Zhou ZR, Wang WW, Li Y, et al. In-depth mining of clinical data: the construction of clinical prediction model with R. Ann Transl Med 2019;7:796.

15. Kim KA, Kim MJ, Jeon HM, et al. Prediction of microvascular invasion of hepatocellular carcinoma: usefulness of peritumoral hypointensity seen on gadoxetate disodium-enhanced hepatobiliary phase images. J Magn Reson Imaging 2012;35:629-34.

16. Kamath A, Roudenko A, Hecht E, et al. CT/MR LIRADS 2018: clinical implications and management recommendations. Abdom Radiol (NY) 2019;44:1306-22.

17. Lee SM, Lee JM, Ahn SJ, et al. LI-RADS Version 2017 versus Version 2018: Diagnosis of Hepatocellular Carcinoma on Gadoxetate Disodium-enhanced MRI. Radiology 2019;292:655-63.

18. Zeng C, Thomas DC, Lewinger JP. Incorporating prior knowledge into regularized regression. Bioinformatics 2021;37:514-21.

19. Goeman JJ. L1 penalized estimation in the Cox proportional hazards model. Biom J 2010;52:70-84.

20. Uno H, Cai T, Pencina MJ, et al. On the C-statistics for evaluating overall adequacy of risk prediction procedures with censored survival data. Stat Med 2011;30:1105-17.

21. Iasonos A, Schrag D, Raj GV, et al. How to build and interpret a nomogram for cancer prognosis. J Clin Oncol 2008;26:1364-70.

22. Balachandran VP, Gonen M, Smith JJ, et al. Nomograms in oncology: more than meets the eye. Lancet Oncol 2015;16:e173-80.

23. Vickers AJ, Cronin AM, Elkin EB, et al. Extensions to decision curve analysis, a novel method for evaluating diagnostic tests, prediction models and molecular markers. BMC Med Inform Decis Mak 2008;8:53.

24. Gigante E, Ronot M, Bertin C, et al. Combining imaging and tumour biopsy improves the diagnosis of combined hepatocellular-cholangiocarcinoma. Liver Int 2019;39:2386-96.

25. Chernyak V, Fowler KJ, Kamaya A, et al. Liver Imaging Reporting and Data System (LI-RADS) Version 2018: Imaging of Hepatocellular Carcinoma in At-Risk Patients. Radiology 2018;289:816-30.

26. Wakizaka K, Yokoo H, Kamiyama T, et al. Clinical and pathological features of combined hepatocellularcholangiocarcinoma compared with other liver cancers. J Gastroenterol Hepatol 2019;34:1074-80.

27. Xue R, Chen L, Zhang C, et al. Genomic and Transcriptomic Profiling of Combined Hepatocellular and Intrahepatic Cholangiocarcinoma Reveals Distinct Molecular Subtypes. Cancer Cell 2019;35:932-947.e8.

28. Leoni S, Sansone V, Lorenzo S, et al. Treatment of Combined Hepatocellular and Cholangiocarcinoma. Cancers (Basel) 2020;12:794. 
29. Roayaie S, Blume IN, Thung SN, et al. A system of classifying microvascular invasion to predict outcome after resection in patients with hepatocellular carcinoma. Gastroenterology 2009;137:850-5.

30. Andreana L, Burroughs AK. Treatment of early hepatocellular carcinoma: How to predict and prevent recurrence. Dig Liver Dis 2010;42 Suppl 3:S249-57.

31. Erstad DJ, Tanabe KK. Prognostic and Therapeutic Implications of Microvascular Invasion in Hepatocellular Carcinoma. Ann Surg Oncol 2019;26:1474-93.

32. Kang Y, Massagué J. Epithelial-mesenchymal transitions: twist in development and metastasis. Cell 2004;118:277-9.

33. Villanueva A, Hoshida Y, Battiston C, et al. Combining clinical, pathology, and gene expression data to predict recurrence of hepatocellular carcinoma. Gastroenterology 2011;140:1501-12.e2.

34. Kim AY, Sinn DH, Jeong WK, et al. Hepatobiliary MRI as novel selection criteria in liver transplantation for hepatocellular carcinoma. J Hepatol 2018;68:1144-52.

35. Yamashita Y, Tsuijita E, Takeishi K, et al. Predictors for microinvasion of small hepatocellular carcinoma $\leq 2 \mathrm{~cm}$. Ann Surg Oncol 2012;19:2027-34.

36. Zhao H, Chen C, Gu S, et al. Anatomical versus nonanatomical resection for solitary hepatocellular carcinoma without macroscopic vascular invasion: A propensity score matching analysis. J Gastroenterol Hepatol 2017;32:870-8.

37. Wang $T$, Yang $X$, Tang $H$, et al. Integrated nomograms to predict overall survival and recurrence-free survival in patients with combined hepatocellular cholangiocarcinoma (cHCC) after liver resection. Aging (Albany NY) 2020;12:15334-58.

38. Wang X, Wang W, Ma X, et al. Combined hepatocellularcholangiocarcinoma: which preoperative clinical data and conventional MRI characteristics have value for the prediction of microvascular invasion and clinical significance? Eur Radiol 2020;30:5337-47.

39. Zheng BH, Ma JQ, Tian LY, et al. The distribution of immune cells within combined hepatocellular carcinoma and cholangiocarcinoma predicts clinical outcome. Clin

Cite this article as: Wang Y, Zhou CW, Zhu GQ, Li N, Qian XL, Chong HH, Yang C, Zeng MS. A multidimensional nomogram combining imaging features and clinical factors to predict the invasiveness and metastasis of combined hepatocellular cholangiocarcinoma. Ann Transl Med 2021;9(20):1518. doi: 10.21037/atm-21-2500
Transl Med 2020;10:45-56.

40. Ma W, Zhang P, Qi J, et al. Prognostic value of platelet to lymphocyte ratio in hepatocellular carcinoma: a metaanalysis. Sci Rep 2016;6:35378.

41. Zhang F, Lu S, Tian M, et al. Albumin-to-Alkaline Phosphatase Ratio is an Independent Prognostic Indicator in Combined Hepatocellular and Cholangiocarcinoma. J Cancer 2020;11:5177-86.

42. Park SH, Lee SS, Yu E, et al. Combined hepatocellularcholangiocarcinoma: Gadoxetic acid-enhanced MRI findings correlated with pathologic features and prognosis. J Magn Reson Imaging 2017;46:267-80.

43. Fowler KJ, Sheybani A, Parker RA 3rd, et al. Combined hepatocellular and cholangiocarcinoma (biphenotypic) tumors: imaging features and diagnostic accuracy of contrast-enhanced CT and MRI. AJR Am J Roentgenol 2013;201:332-9.

44. Yang L, Gu D, Wei J, et al. A Radiomics Nomogram for Preoperative Prediction of Microvascular Invasion in Hepatocellular Carcinoma. Liver Cancer 2019;8:373-86.

45. Xu X, Zhang HL, Liu QP, et al. Radiomic analysis of contrast-enhanced CT predicts microvascular invasion and outcome in hepatocellular carcinoma. J Hepatol 2019;70:1133-44.

46. Dageforde LA, Vachharajani N, Tabrizian P, et al. MultiCenter Analysis of Liver Transplantation for Combined Hepatocellular Carcinoma-Cholangiocarcinoma Liver Tumors. J Am Coll Surg 2021;232:361-71.

47. Groeschl RT, Turaga KK, Gamblin TC. Transplantation versus resection for patients with combined hepatocellular carcinoma-cholangiocarcinoma. J Surg Oncol 2013;107:608-12.

48. Iguchi T, Shirabe K, Aishima S, et al. New Pathologic Stratification of Microvascular Invasion in Hepatocellular Carcinoma: Predicting Prognosis After Living-donor Liver Transplantation. Transplantation 2015;99:1236-42.

49. Le Roy B, Gelli M, Pittau G, et al. Neoadjuvant chemotherapy for initially unresectable intrahepatic cholangiocarcinoma. Br J Surg 2018;105:839-47. 
A $\quad \begin{array}{llllllllllllllllllll}41 & 40 & 39 & 39 & 38 & 35 & 34 & 34 & 29 & 18 & 14 & 3 & 1\end{array}$

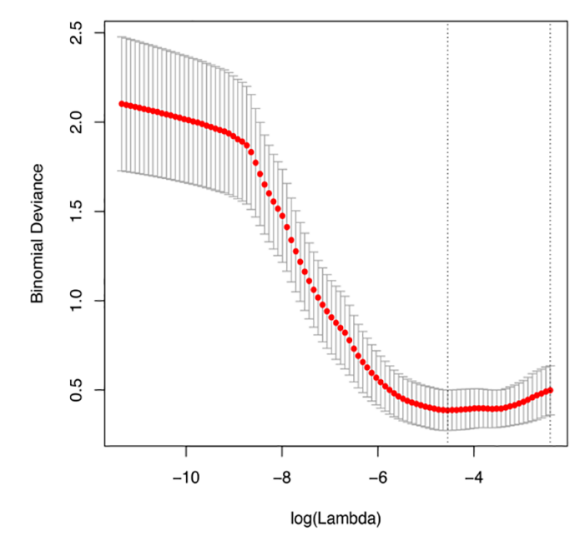

B

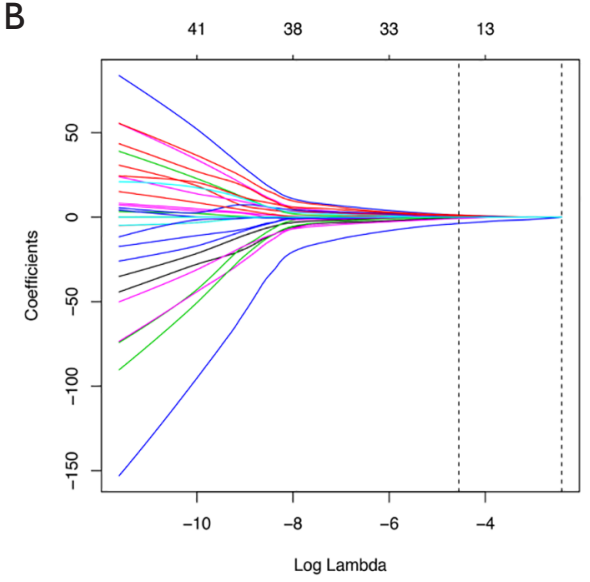

C

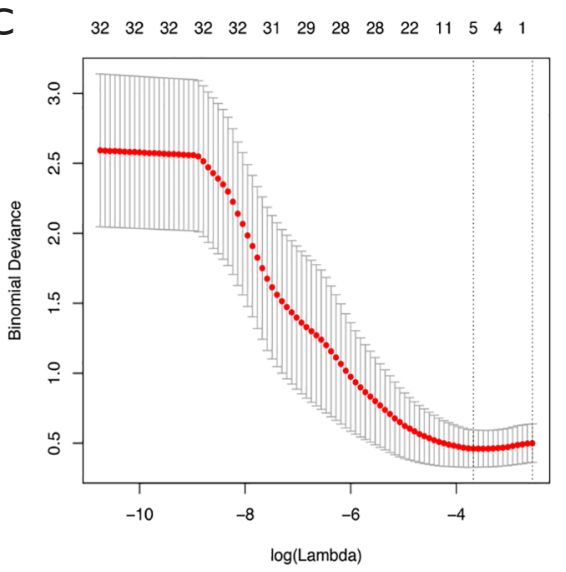

D

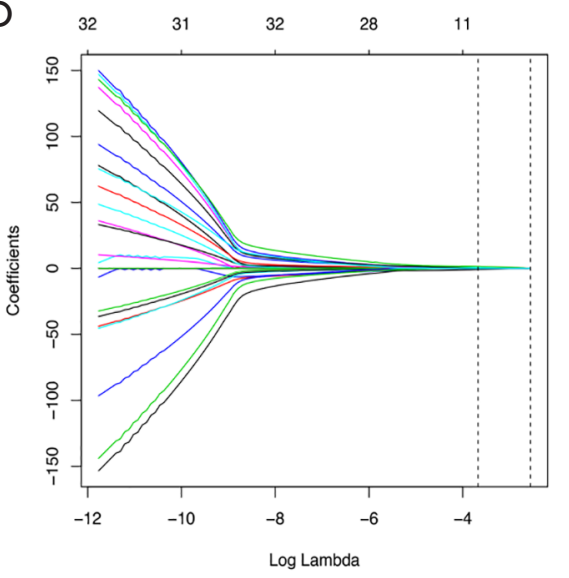

Figure S1 Selection of imaging (A,B) and clinical features (C,D) using the LASSO logistic regression algorithm for prediction of LNM. The penalization coefficient $\lambda$ in the LASSO model was tuned using 10-fold cross-validation and minimum criterion for presence of LNM. The lower $\mathrm{X}$-axis shows $\log (\lambda)$, while the top $\mathrm{X}$-axis indicates the number of predictors for the given $\log (\lambda)$. The Y-axis indicates binomial deviance. Red dots represent average misclassification errors for every model with given $\lambda$, and the vertical bars indicate the upper and lower values of the misclassification errors. The vertical black lines define the optimal $\lambda$, which provides its best fit to the data. As a result, an optimal $\lambda$ of 0.017 , with $\log (\lambda)=-4.085$, was selected for LNM prediction using clinical features, and an optimal $\lambda$ of 0.025 , with $\log (\lambda)$ $=-3.674$, was selected for LNM prediction using imaging features. LASSO, least absolute shrinkage, and selection operator; LNM, lymph node metastasis.

Table S1 Formulas for imaging scores and clinical scores in the LASSO models for MVI, satellite nodules and LNM status

\begin{tabular}{ll}
\hline Group & Formula \\
\hline Imaging scores for & $-0.25394 \times$ shape: globular $+0.59647 \times$ targocid restriction: absent $-0.069 \times$ arterial enhancement pattern: global \\
MVI status & $-0.76723 \times$ peritumoral enhancement pattern: none $+0.10008 \times$ peritumoral enhancement pattern: wedge -0.68747 \\
& $\times$ washout: absent $-0.36876 \times$ false capsule: none $+0.05932 \times$ false capsule: incomplete $-0.06953 \times$ bile duct \\
& dilation: absent $-0.09913 \times$ surface retraction: absent $+0.1107 \times$ nodule in nodule architecture: absent $-0.43829 \times$ \\
& LR-RADS categorization: LR-3 $+0.88054 \times$ LR-RADS categorization: LR-TIV -0.036
\end{tabular}

LASSO, least absolute shrinkage, and selection operator; MVI, microvascular invasion; LNM, lymph node metastasis; LI-RADS, Liver Imaging Reporting and Data System; PLR, platelet to lymphocyte ratio; AAPR, albumin-to-alkaline phosphatase ratio; AST, aspartate aminotransferase; CA 19-9, carbohydrate antigen 19-9. 


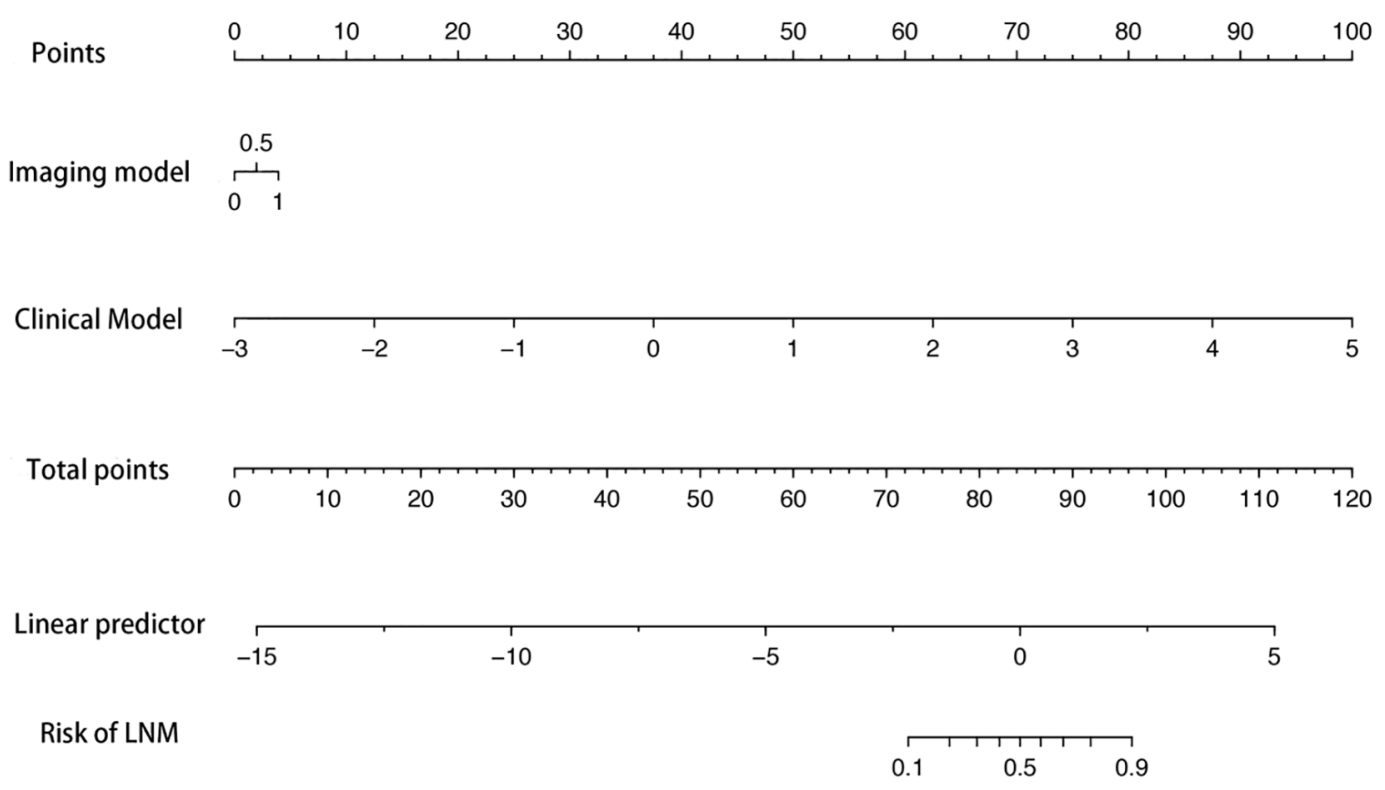

Figure S2 Nomogram for predicting the presence of LNM. Points are assigned for clinical model and imaging model for predicting the presence of LNM. LNM, lymph node metastasis.

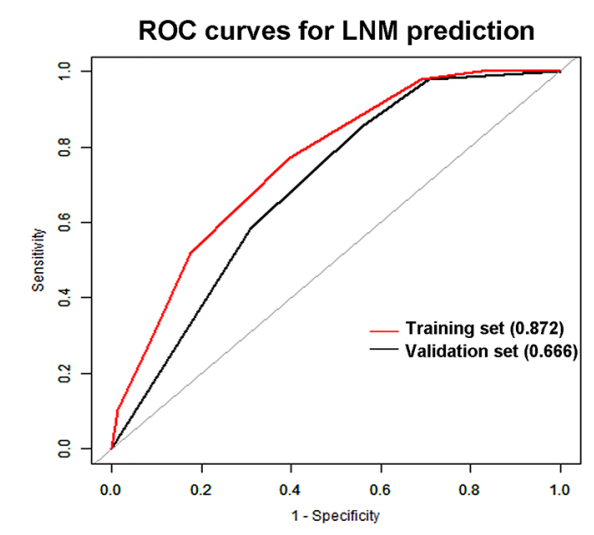

Figure S3 Receiver operating curve for predicting the LNM in the training set (A) and validation set (B) set using the combination nomogram. ROC, receiver operating characteristic; LNM, lymph node metastasis.

A

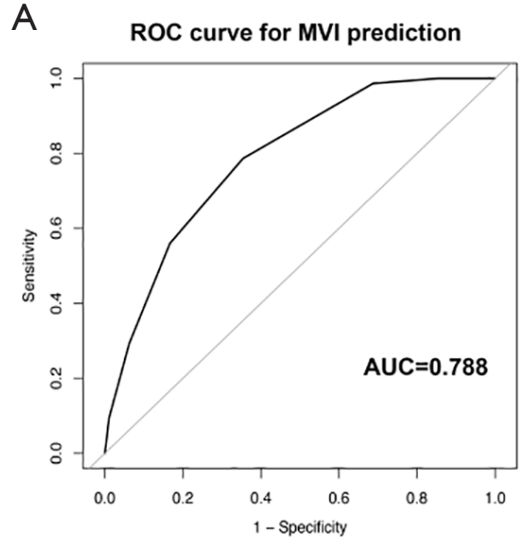

B ROC curve for satellite nodules prediction

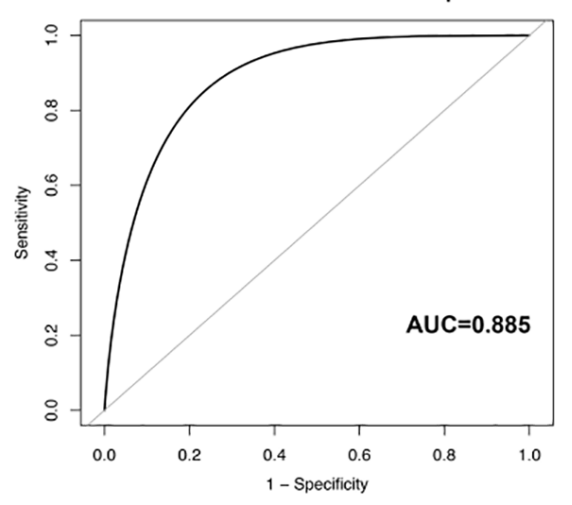

C ROC curve for LNM prediction

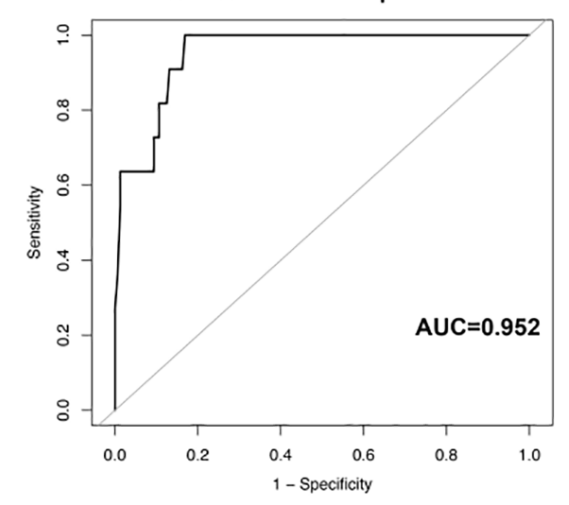

Figure S4 Receiver operating curve for predicting the MVI (A), the presence of satellite nodules (B) and LNM (C) in the CHCC-CCA subtype. ROC, receiver operating characteristic; MVI, microvascular invasion, LNM, lymph node metastasis; CHCC-CCA, combined hepatocellular cholangiocarcinoma. 

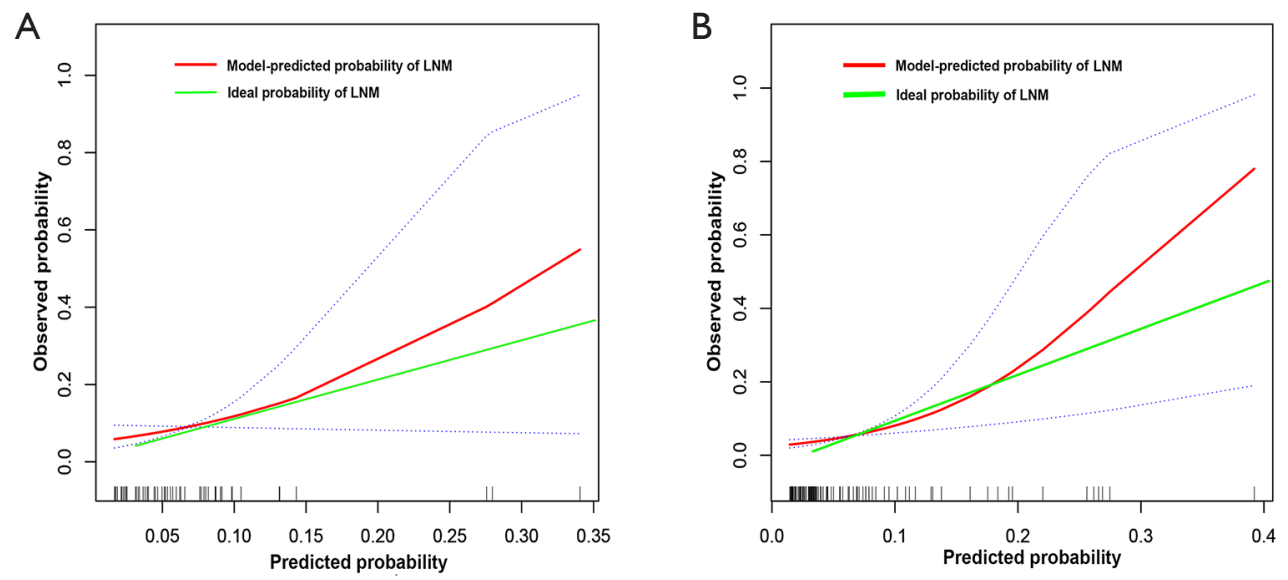

Figure S5 The calibration curves of the combination nomogram for prediction of LNM in the training set (A) and validation set (B). LNM, lymph node metastasis.

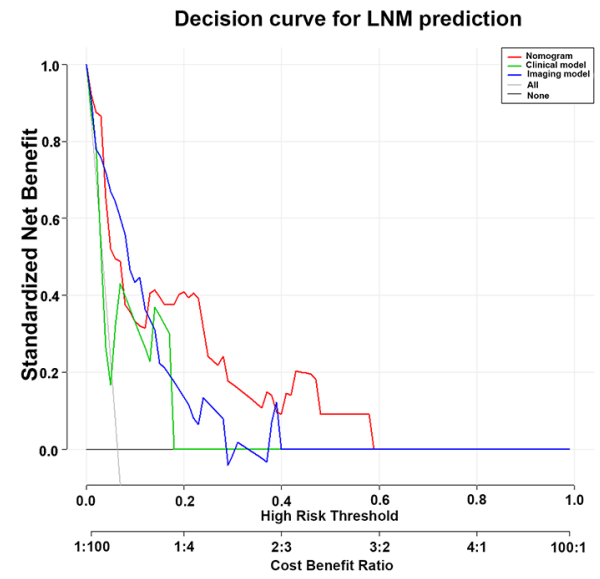

Figure S6 The decision curves of the combination nomogram, clinical model, and imaging model for prediction of LNM in overall patients. Vertical axis: the net benefit; horizontal axis: the threshold probability at a range of 0.0 to 1.0 . The gray line represents the decision curve of the assumption that all patients suffer from LNM; the black line represents the decision curve of the assumption that no patients suffer from LNM. ROC, receiver operating characteristic; MVI, microvascular invasion; LNM, lymph node metastasis. 